\title{
Comparing UV/EUV line parameters and magnetic field in a quiescent prominence with tornadoes
}

\author{
P. J. Levens ${ }^{1}$, N. Labrosse ${ }^{1}$, B. Schmieder ${ }^{2}$, A. López Ariste ${ }^{3,4}$, and L. Fletcher ${ }^{1}$ \\ 1 SUPA School of Physics and Astronomy, University of Glasgow, Glasgow, G12 8QQ, UK \\ e-mail: p.levens.1@research.gla.ac.uk \\ 2 LESIA, Observatoire de Paris, PSL Research University, CNRS, Sorbonne Universités, UPMC Univ. Paris 06, Univ. Paris Diderot, \\ Sorbonne Paris Cité, 5 place Jules Janssen, 92195 Meudon, France \\ 3 IRAP - CNRS UMR 5277, 14 Av. E. Belin, 31400 Toulouse, France \\ ${ }^{4}$ Université de Toulouse, UPS-OMP, Institut de Recherche en Astrophysique et Planétologie, 31400 Toulouse, France
}

Received 17 March 2017 / Accepted 11 August 2017

\begin{abstract}
Context. Understanding the relationship between plasma and the magnetic field is important for describing and explaining the observed dynamics of solar prominences.

Aims. We determine if a close relationship can be found between plasma and magnetic field parameters, measured at high resolution in a well-observed prominence.

Methods. A prominence observed on 15 July 2014 by the Interface Region Imaging Spectrograph (IRIS), Hinode, the Solar Dynamics Observatory (SDO), and the Télescope Héliographique pour l'Étude du Magnétisme et des Instabilités Solaires (THEMIS) is selected. We perform a robust co-alignment of data sets using a $2 \mathrm{D}$ cross-correlation technique. Magnetic field parameters are derived from

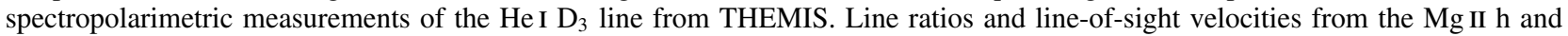
$\mathrm{k}$ lines observed by IRIS are compared with magnetic field strength, inclination, and azimuth. Electron densities are calculated using Fe XII line ratios from the Hinode Extreme-ultraviolet Imaging Spectrometer, which are compared to THEMIS and IRIS data.

Results. We find $\mathrm{Mg} \mathrm{II} \mathrm{k/h}$ ratios of around 1.4 everywhere, similar to values found previously in prominences. Also, the magnetic field is strongest $(\sim 30 \mathrm{G})$ and predominantly horizontal in the tornado-like legs of the prominence. The $\mathrm{k}_{3}$ Doppler shift is found to be between $\pm 10 \mathrm{~km} \mathrm{~s}^{-1}$ everywhere. Electron densities at a temperature of $1.5 \times 10^{6} \mathrm{~K}$ are found to be around $10^{9} \mathrm{~cm}^{-3}$. No significant correlations are found between the magnetic field parameters and any of the other plasma parameters inferred from spectroscopy, which may be explained by the large differences in the temperatures of the lines used in this study.

Conclusions. This is the first time that a detailed statistical study of plasma and magnetic field parameters has been performed at high spatial resolution in a prominence. Our results provide important constraints on future models of the plasma and magnetic field in these structures.
\end{abstract}

Key words. Sun: filaments, prominences - Sun: UV radiation - Sun: magnetic fields - Sun: atmosphere

\section{Introduction}

Solar "tornadoes" in prominences have recently been the focus of several papers debating their true nature. With the launch of the Solar Dynamics Observatory and its high resolution Atmospheric Imaging Assembly (SDO, AIA; Lemen et al. 2012) imager, several authors (Su et al. 2012; Li et al. 2012; Panesar et al. 2013; Wedemeyer et al. 2013) noted tornado-like structures in the solar atmosphere. These were seen as dark columns of apparently rotating material, which were absorbing background coronal emission. Some authors (e.g. Panasenco et al. 2014) argued that the observed motions can be interpreted as oscillations in the plane of the sky.

More recent observations with spectroscopic instruments have allowed measurements of the line-of-sight velocities and several plasma diagnostics in these structures. Su et al. (2014) and Levens et al. (2015) used the Extreme-ultraviolet Imaging Spectrometer (EIS; Culhane et al. 2007) on the Hinode satellite (Kosugi et al. 2007) to measure Doppler velocities in a tornado, finding an anti-symmetric pattern along the axis of the column at plasma temperatures above $10^{6} \mathrm{~K}$. This pattern has also been seen in $\mathrm{H} \alpha$ (Wedemeyer et al. 2013) and He I $10830 \AA$
(Orozco Suárez et al. 2014), however it is unclear whether these observations are really showing rotation. Other observations have cast doubt on the presence of long-term rotational patterns. Schmieder et al. (2017b) show that Doppler patterns suggestive of rotational motions over a short period of time disappear on longer timescales. These authors detected changing patterns of Doppler shifts, indicating that we are seeing oscillations with periods of the order of 40 to $60 \mathrm{~min}$.

Schmieder et al. (2017a) used the high spectral and spatial resolution of the Interface Region Imaging Spectrograph satellite (IRIS; De Pontieu et al. 2014) to reconstruct the 3D trajectories of plasma "blobs" in a helical prominence. Despite the plasma tracing highly curved paths in the plane of the sky, it was found that the actual trajectories of the plasma were along horizontal paths, indicating that the apparent plasma motion can differ significantly from the real motion.

Some work has also now been done on measuring the magnetic field in tornado-like prominences (Levens et al. 2016a,b; Martinez Gonzalez et al. 2016). Levens et al. (2016a,b) found that the field in these tornadoes is largely horizontal, parallel to the limb, with field strengths of between 10 and $50 \mathrm{G}$. 

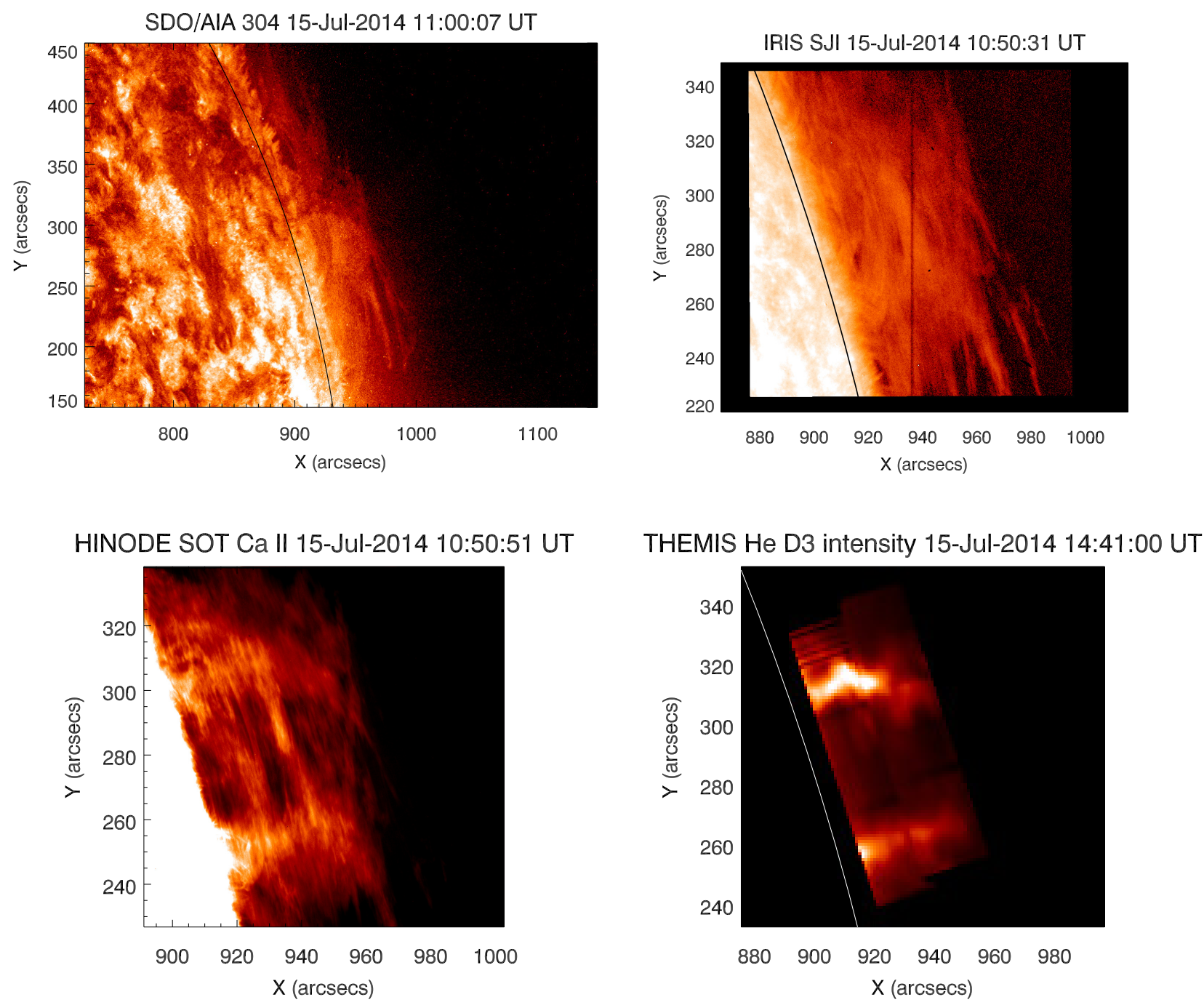

Fig. 1. Observations by several instruments on 15 July 2014. Clockwise from upper left: AIA observation in $304 \AA$ A IRIS SJI map using the Mg II filter. THEMIS map, made using the two rasters obtained on that day in the He I $\mathrm{D}_{3}$ line (rotated and aligned with the SOT image). Hinode SOT map in Ca II.

This does not support the twisted models suggested in Su et al. (2014) or as modelled by Luna et al. (2015), rather suggesting the plasma is in a horizontal field with dips over parasitic polarities on the solar surface (Aulanier \& Démoulin 1998). Martinez Gonzalez et al. (2016) find a mix of possible field orientations, which they interpret as evidence for a twisted field.

The link, however, between the magnetic field and the plasma parameters that are measurable remains unclear. In this work we aim to study the magnetic field parameters, as measured by the Télescope Héliographique pour l'Étude du Magnétisme et des Instabilités Solaires (THEMIS) instrument in the Canary Islands, in comparison with plasma parameters from the $\mathrm{Mg} \mathrm{II} \mathrm{h}$ and $\mathrm{k}$ lines, measured by IRIS. We study the magnetic field strength and orientation, and look for correlations with characteristics derived from $\mathrm{Mg}$ II line profiles (velocities, optical thickness, and intensity ratios, amongst others).

We introduce the observations and the instruments used in Sect. 2, as well as outlining the diagnostic parameters that can be derived from these observations. Section 3 details the methods for co-aligning the various data sets that are used. In Sect. 4 we present our results and correlation plots between parameters available from IRIS and THEMIS. Section 5 contains results and correlations between the electron density, measured by Hinode EIS and magnetic field parameters from THEMIS. We also look for correlations between EIS and IRIS, which are shown in Sect. 6. Section 7 includes our concluding remarks on this work.

\section{Observations and diagnostic parameters available}

The prominence observed on 15 July 2014 above the western solar limb was seen brightly in emission by AIA in the $304 \AA$ passband (Fig. 1), whereas in the coronal filters (e.g. $171 \AA$, Fig. 2) we see two columns of dark material, which are absorbing the background emission. The emission seen in the AIA image of Fig. 1 is dominated by the He II doublet at $303.78 \AA$; these are optically thick emission lines formed mostly by resonant scattering of emission from the solar surface below the prominence (Labrosse \& Gouttebroze 2001). The $171 \AA$ images from AIA, however, are dominated by emission from Fe IX, formed at around $1 \mathrm{MK}$. The dark columns are comprised mostly of cooler material, hydrogen and helium, that absorbs the emission coming from the corona behind. These columns have been observed 


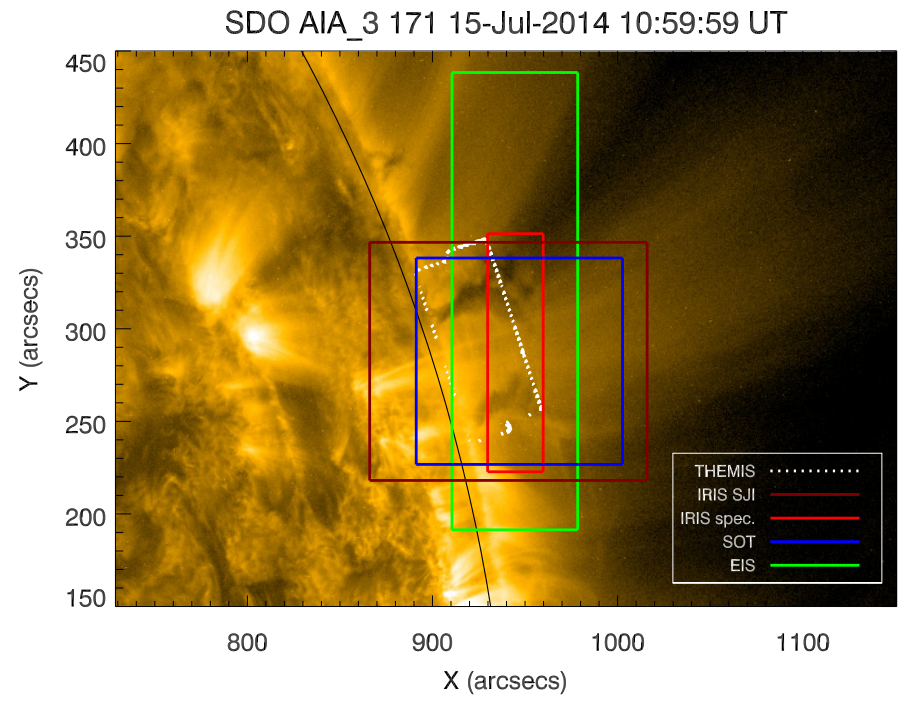

Fig. 2. AIA observation from 15 July 2014 in $171 \AA$ A. The coloured boxes indicate the fields of view of the other instruments used in this study.

to display oscillatory behaviour when viewed over time ( $\mathrm{Su}$ et al. 2012, 2014; Levens et al. 2016a), which has been interpreted as tornado-like rotation, so here we refer to them as "tornadoes".

Plasma parameters for this prominence were explored in Levens et al. (2016a, herein Paper I), but here we aim to take a deeper look at the diagnostics available. Full details of the observations and more about the dynamics of the prominence are covered in Paper I.

\subsection{IRIS}

\subsubsection{Observations}

The IRIS observations from the 15 July 2014 consisted of 16step coarse rasters between 08:00 UT and 11:00 UT. Spatial scale is $0.167^{\prime \prime}$ in $y$, with an actual spatial resolution of around $0.35^{\prime \prime}$, and a step size of $2^{\prime \prime}$ in $x$. Exposure time was $5.4 \mathrm{~s}$ per slit position, giving a raster cadence of $86.4 \mathrm{~s}$. These rasters contained both near-ultraviolet (NUV, 2783-2834 ̊) and farultraviolet (FUV, 1332-1348 $\AA$ and 1390-1406 $\AA$ ) lines. In this paper we focus on the NUV channel, namely the Mg II $\mathrm{h}$ and $\mathrm{k}$ lines at $2803.5 \AA$ and $2796.35 \AA$ respectively. Raw data is calibrated to level-2 (De Pontieu et al. 2014), where dark current subtraction, geometrical correction, and flat field correction have each been accoundted for.

Slit-jaw images (SJI) were taken in the broadband filters centred on $2976 \AA$ (Mg II) and $1330 \AA$ (C II). The cadence for SJI was $11 \mathrm{~s}$, and the field of view (FOV) covered 119" $\times 119^{\prime \prime}$.

\subsubsection{Plasma diagnostics}

The Mg II h and k lines are optically thick in prominences, evident from observed centrally-reversed profiles (Paper I). However, they are not always reversed (Vial 1982; Vial et al. 2016; Schmieder et al. 2014), often showing non-reversed, singlepeaked profiles. This behaviour can be explained by different physical conditions in different regions of the prominence (Heinzel et al. 2014, 2015). In the prominence observed on 15 July 2014, we find a mixture of reversed and non-reversed profiles (see Fig. 3 and Paper I).
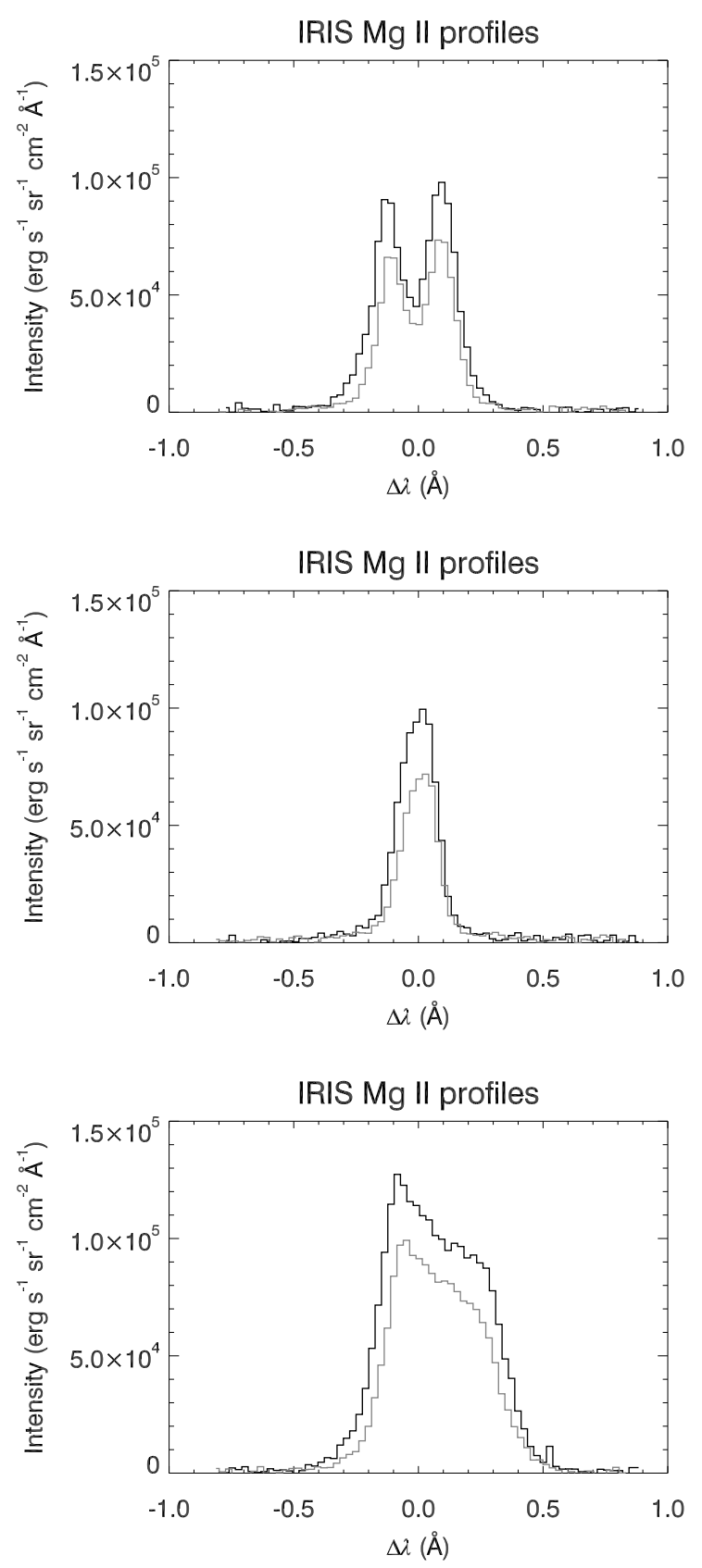

Fig. 3. Example $\mathrm{Mg}$ II $\mathrm{k}$ and $\mathrm{h}$ line profiles from the prominence of 15 July 2014. Each is taken from the raster that started at 10:21 UT. Shown are both $\mathrm{k}$ (black lines) and h (grey lines) profiles. Top: reversed profiles. Middle: single-peaked profiles. Bottom: complex profiles.

As is usually done, we call an Mg II line profile reversed when there are two distinct peaks in the line, known as the $\mathrm{k}_{2}$ or $h_{2}$ peaks for the $k$ and $h$ lines respectively, surrounding a region of lower intensity (the line core, known as $\mathrm{k}_{3}$ and $\mathrm{h}_{3}$ respectively). Typical reversed profiles can be seen in Fig. 3 (top). By single-peaked profiles, we refer to line profiles showing only one peak, which is close to the line centroid (as would be determined, for instance, by an approximate Gaussian fit). Typical examples of single-peaked profiles are shown in the middle panel of Fig. 3. A large fraction of observed profiles do not fit in either of these two categories, however, and we therefore call them complex profiles (Fig. 3 bottom). Complex profiles may have no clear central reversal, or a broad, flat line core, or may 
have just one peak but away from the line centre (at a distance $>0.04 \AA$ ), or show several intensity peaks.

The existence of such complex profiles has two possible explanations. The first is as in Schmieder et al. (2014), where numerous threads with different velocity components along the line of sight create multiple-component profiles. In this scenario, single-peaked Gaussian profiles blend together when observed by IRIS. Schmieder et al. (2014) found, using multiple narrow Gaussians, that there were observable flows of up to $80 \mathrm{~km} \mathrm{~s}^{-1}$ in a quiescent prominence. A second possibility is that of a reversed profile with one of the peaks missing. This could be due to seeing multiple optically thick threads with different velocity components along the line of sight. This model was explored by Gunár et al. (2008) to explain asymmetrical Lyman line profiles, and by Labrosse \& Rodger (2016) for helium lines in prominences. It has been shown that a combination of a number of reversed profiles with different line-of-sight velocities can create an emergent reversed line profile with one peak missing (Gunár et al. 2008). This could occur due to the optical thickness difference across the $\mathrm{Mg}$ II $\mathrm{h}$ and $\mathrm{k}$ line profiles, and the differences in where the component parts of those profiles are formed in the prominence, under the assumption of a multithread model. The complex profiles could then be explained by "stationary" line wings and $\mathrm{k}_{2}$ peaks resulting from an integration along the line of sight of several threads, and a shifted $\mathrm{k}_{3}$ core due to the motion of the frontmost thread. This, then, absorbs emission in one of the $\mathrm{k}_{2}$ regions, reducing the emission from that peak. In this data set there do not appear to be highly Doppler shifted profiles, as was discussed in Paper I, so we do not believe that these profiles are a blend of multiple line-ofsight components. Also discussed in Paper I was the high optical thickness of the $\mathrm{Mg}$ II resonance lines in this prominence, apparent from comparing the levels of reversal seen to the models of Heinzel et al. (2014). This points towards the second scenario as the most likely explanation for the "complex" profiles seen here. A further investigation into these profiles may be useful, as they appear to be common in prominences. Regardless of the formation mechanism, we need to characterise these profiles in order to use them in this analysis.

To make an estimate of the level of reversal in the complex profiles, we make the assumption that the centroid of the line, calculated from the moments of the distribution, is the $\mathrm{k}_{3}$ location, and the maximum intensity of the profile corresponds to the $\mathrm{k}_{2}$ peak. We use an algorithm presented in Waller et al. (in prep.) to automatically measure the Mg II line profile characteristics. Complex profiles are found automatically by comparing the centroid of the distribution to the position of the peak of the distribution. For a truly single-peaked profile, these two values should not be more than a few $m \AA$ apart. However, for a complex profile they are more separated. We set a limit of $0.04 \AA$ on the distance between the positions of the line centroid and the peak for a profile to be classed as complex.

\subsection{THEMIS}

\subsubsection{Observations}

THEMIS ran two raster studies using the Multi Raies (MTR) spectrograph (López Ariste et al. 2000) on 15 July 2014. The first raster started at 14:41 UT and the second at 16:55 UT, each taking $30 \mathrm{~min}$ to complete, putting these rasters after the observations made by IRIS and Hinode. However, the conditions of the prominence and tornadoes did not change significantly in the interval between the sets of observations. Therefore, we can assume that parameters obtained in the morning are not dissimilar to those obtained in the afternoon by THEMIS, but we must keep in mind that there is this temporal discrepancy between the space-based and ground-based observations. In both rasters the slit of the spectrograph was orientated parallel to the limb, and each consisted of 30 slit positions separated by $2^{\prime \prime}$. Original spatial pixel size is $0.23^{\prime \prime}$, but data used here has been binned to have square pixels of $1^{\prime \prime} \times 1$ ". Exposure time is $1.5 \mathrm{~s}$ per Stokes parameter, with six Stokes parameters per cycle, and ten repeats of each cycle at each slit position to increase signal-to-noise ratio (SNR). Paper I contains further details of the study.

The THEMIS MTR instrument is a spectropolarimeter, giving observations of the four Stokes parameters $(I, Q, U$, and $V$ ) using the $\mathrm{He} \mathrm{I}_{3}$ line. The data was handled using the DeepStokes procedure (López Ariste et al. 2009), then the Stokes profiles were treated using the code of López Ariste \& Casini (2002) and Casini et al. (2003), which is based on principal component analysis. After this treatment, the resulting observed profiles are compared to those in a model database of over 90000 profiles, generated for He I accounting for both the Hanle and Zeeman effects (López Ariste \& Casini 2002). The most similar profile in the database to the observed profile is taken to be the solution, which gives us information about the magnetic field strength and orientation at each pixel. Again, more details are found in Paper I and references therein.

\subsubsection{Plasma diagnostics}

The main parameters that THEMIS provides are the magnetic field strength and the orientation of the field, namely the field inclination and azimuth. We also have the intensity image in $\mathrm{He} \mathrm{I} \mathrm{D}_{3}$ (Fig. 1, lower right), which gives us information about the spatial structure of the prominence in that wavelength. Figure 4 shows the magnetic field map for the tornadoes on 15 July 2014.

\subsection{Hinode}

\subsubsection{Observations}

Hinode was observing co-temporally with IRIS, with both the Solar Optical Telescope (SOT; Suematsu et al. 2008; Tsuneta et al. 2008) and EIS aquiring data.

During this study, SOT observed using the CaII $H$ filter centred at $3968.5 \AA$, with images taken with a 30 s cadence and a pixel size of $0.109^{\prime \prime}$. SOT observed from 10:21 UT until 11:06 UT, covering the last part of the IRIS observing time. The SOT FOV was $112^{\prime \prime} \times 112^{\prime \prime}$ and is shown as a blue box in Fig. 2. Raw SOT data is calibrated to level-1 using the standard SolarSoftWare (SSW) routine (fg_prep).

EIS ran a 35-step raster using the $2^{\prime \prime}$ slit, covering a $70^{\prime \prime} \times$ $248^{\prime \prime}$ area (green box, Fig. 2). The study used was madj_qs, which has an exposure time of $50 \mathrm{~s}$, meaning that a full raster is made in around $30 \mathrm{~min}$. Only one raster was achieved during the observing time, starting at 10:34 UT and ending at 11:06 UT. The study madj_qs observes eleven wavelength channels in both the long-wavelength CCD and short-wavelength CCD of EIS, allowing access to spectral lines formed at a range of plasma temperatures from around $8 \times 10^{5} \mathrm{~K}$ to more than $2 \times 10^{6} \mathrm{~K}$. EIS data is acquired at level-0, and hot (and warm) pixels, dark currents, and cosmic-ray hits are removed using the SSW routine eis_prep. This routine also performs absolute calibration of the data, leaving it ready for analysis at level-1.

In order to isolate spectral lines from EIS, Gaussian functions are fitted. This allows us to derive plasma parameters at 
THEMIS He D3 field strength 15-Jul-2015 14:41:00 UT

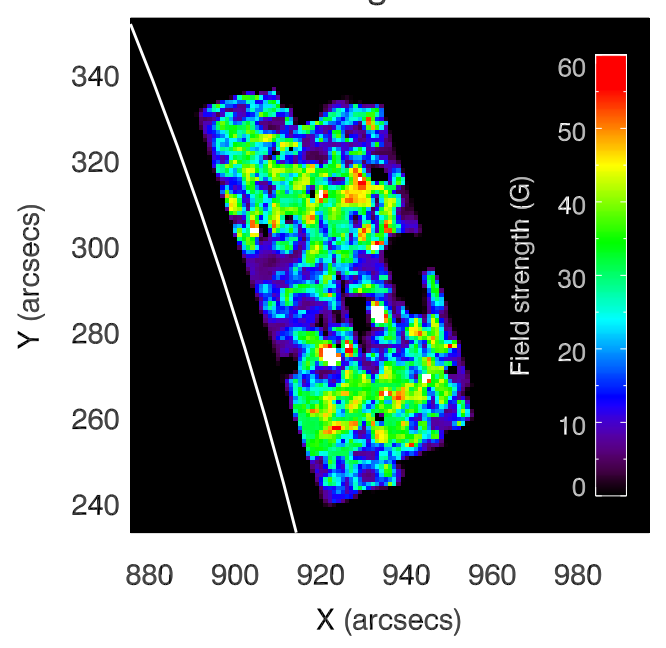

Fig. 4. Magnetic field map for the prominence on 15 July 2014, calculated using the $\mathrm{He} \mathrm{I} \mathrm{D}_{3}$ line from THEMIS. Image is made using both rasters from that day, one starting at 14:41 UT and the second starting at 16:55 UT. Solar limb position is shown in white.

each pixel for each line, creating a series of maps using each Gaussian fit.

\subsubsection{Plasma diagnostics}

SOT brings us intensity images for a spectral window around the $\mathrm{Ca}$ II $\mathrm{H}$ line. These are useful as they provide another opticallythick intensity image that can be used for co-aligning data sets.

EIS provides spectra for a large number of EUV spectral lines from ionised elements. The plasma emitting these lines is mostly optically thin and at coronal temperatures, however, some cooler, optically-thick lines are also observed, such as He II $256 \AA$. Optically-thin lines are fitted with Gaussian profiles. This approximation allows us to derive plasma properties from the Gaussian parameters, such as line-of-sight velocities and line full width at half maximum (FWHM), as well as electron densities when density-sensitive pairs of lines are observed together. This kind of analysis was done on a solar tornado observed by EIS (Su et al. 2014; Levens et al. 2015), where velocities of less than $5 \mathrm{~km} \mathrm{~s}^{-1}$ were found in the tornado, and a split Doppler pattern across the tornado led the authors to conclude that the tornado is rotating around a central axis. Levens et al. (2015) also presented density diagnostics, finding electron densities, $n_{\mathrm{e}}$, of around $10^{9} \mathrm{~cm}^{-3}$ and noting that there appears to be lower electron density in the tornado than in the surrounding corona at a temperature of $1.5 \times 10^{6} \mathrm{~K}$. The analysis done previously on tornadoes with EIS has mostly focused on coronal temperature plasma, as the cooler lines observed by EIS are often difficult to interpret. For example, the He II line is heavily blended with lines formed at a higher temperature, making it difficult to distinguish the cooler component.

\section{Co-alignment of data}

The aim of this work is to do a statistical analysis on a pixel by pixel basis, so we require that the data sets from different instruments are well aligned. Here we use a 2D crosscorrelation method making use of a mean absolute difference (MAD) algorithm in the get_correl_offsets SSW routine. To cross-correlate data sets with different spatial resolutions, it is necessary to reduce the spatial resolutions to that of the lowest resolution image by binning the data. This re-binning is done individually for each pair of images to be co-aligned. The correlations used are described in detail below.

In this analysis we concentrate on one image from each of the data sets. Due to the discrepancies in observing time between space-based and ground-based observatories on this day, we unfortunately cannot have well temporally-aligned data. However, from AIA images and movies, the prominence and tornadoes do not change much over the course of the day. Differential rotation does not have any influence on a prominence over the course of a few hours, so we do not expect it to affect the prominence observed here.

\subsection{IRIS with SDO AIA}

For the co-alignment of the data sets we take the AIA $304 \AA$ image from 11:00 UT as the base image (Fig. 1 top left panel), to line up with the end of the observing time of IRIS and Hinode. All other data sets will eventually be aligned with this image. The prominence appears as an extended structure in both AIA $304 \AA$ images and IRIS Mg II images, with horizontal fine structure. The similarity is due to the fact that the (dominant) He II emission line seen in the AIA $304 \AA$ passband and the Mg II $\mathrm{h}$ and $\mathrm{k}$ lines are both extremely optically thick, with $\tau_{\mathrm{Mg}} \sim \tau_{\mathrm{He}}($ Paper I).

Figure 1 (top right panel) shows the IRIS SJI from 10:51 UT. IRIS passed through the South Atlantic Anomaly at the end of its observing time, so the data after this time was unusable. We use data from the end of the observing time for the space-based telescopes so as to minimise the time between these data and those from THEMIS, which observed in the afternoon. We crosscorrelate the AIA and SJI data, finding that an offset of $x=0.81^{\prime \prime}$ and $y=-1.65^{\prime \prime}$ needs to be applied to the IRIS SJI to bring it in line with the AIA image.

\subsection{Hinode SOT with IRIS}

The Ca II $\mathrm{H}$ line observed by SOT is an optically-thick emission line. However, it has been seen that the $\mathrm{H}$ and $\mathrm{K}$ lines of calcium are not as optically thick as the $\mathrm{h}$ and $\mathrm{k}$ lines of magnesium. This is evident from Fig. 1 (IRIS and SOT images), where we see more of the column-like structure in the prominence in the SOT image, but not in the IRIS image. We note, however, that the SOT images largely still show the horizontal structure common to both IRIS and AIA $304 \AA$ Amages, so we can use these images for the co-alignment of the data set from SOT. The IRIS SJI data has high spatial resolution so it is used to co-align the SOT data. The cross-correlation routine was run on the SOT images and the resulting offset of $x=11.22^{\prime \prime}$ and $y=45.64^{\prime \prime}$ was applied to the SOT images.

\subsection{THEMIS with Hinode SOT}

Aligning THEMIS with the other instruments is more of a challenge than for other data sets, due to the way THEMIS observes. THEMIS observations of prominences are generally done with the slit orientated parallel to the solar limb at a certain position angle (PA), measured anti-clockwise from solar north, and the first slit position is somewhere near the limb. This means that we do not have a traditional "pointing" for the THEMIS maps, and we must make sure that the map is orientated properly before performing any further alignment. To begin with, the THEMIS data is orientated with the slit parallel to the solar limb, and 

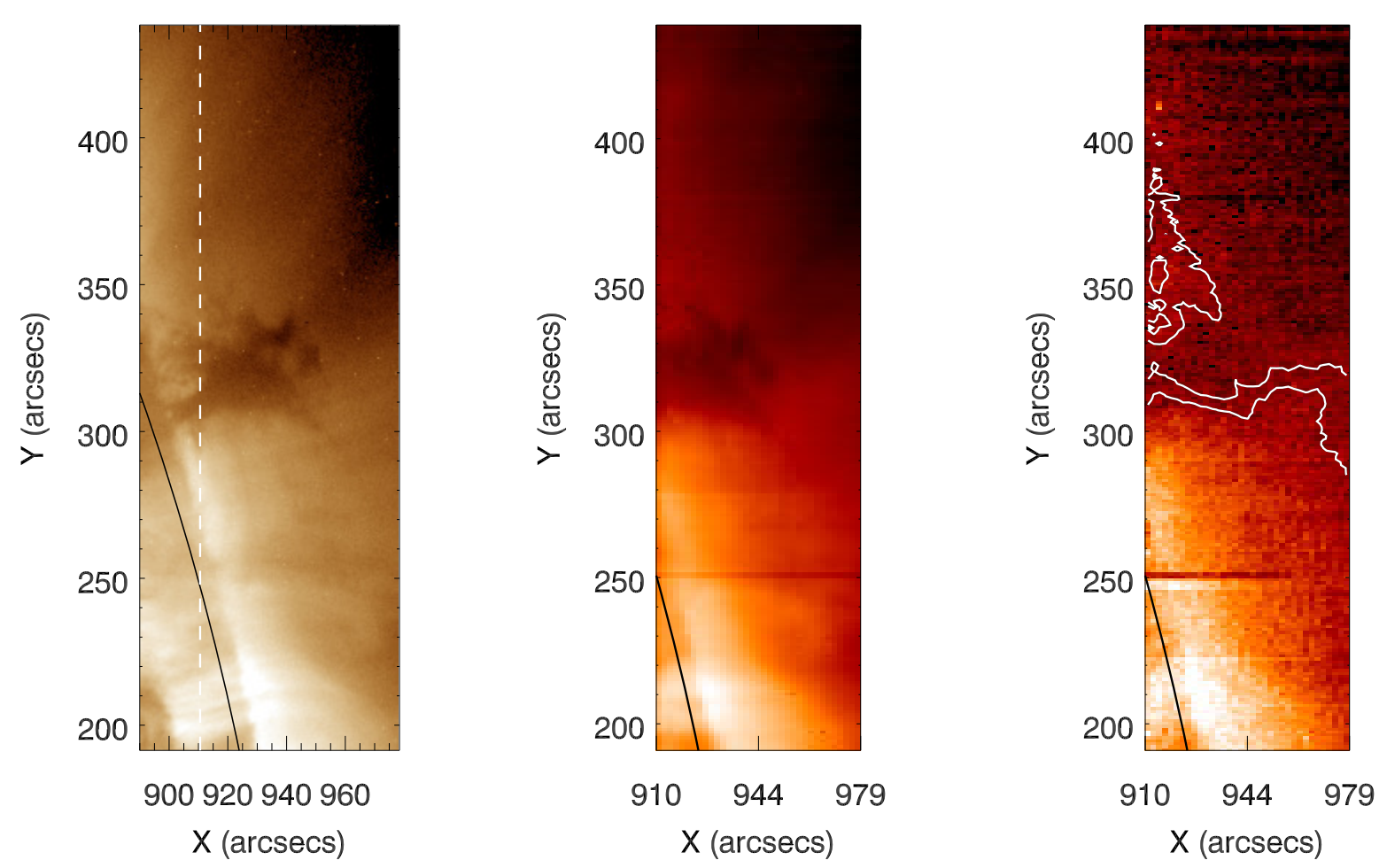

Fig. 5. Maps from the 15 July 2014 for Fe XII lines, showing views of the prominence by AIA and EIS. Left: AIA $193 \AA$ A filter. Vertical dashed line shows the edge of the EIS FOV. Middle: EIS $195.119 \AA$. Right: EIS $195.179 \AA$ with $22 \%$ and $25 \%$ contours of $195.119 \AA$ (this line pair is used for density diagnostics in Sect. 5). The observations were made at 10:34 UT. The dark horizontal features in the EIS maps are artifacts from the EIS detectors.

the slit along the $x$-axis of the resulting raster. On the 15 July 2014 THEMIS made two such images, one at the bottom of the prominence and one near the top. These have been spliced together, as the offset between them in $y$ (height above limb) was known. They are then taken to be one image for the day in this analysis. To orientate these images we rotate the THEMIS map by $360^{\circ}$ minus the PA at which the prominence was observed, which on the 15 July 2014 was PA $=288^{\circ}$, giving an image roll of $72^{\circ}$. The rotated THEMIS image can be seen in Fig. 1 (bottom right panel). The centre of this rotated map was shifted manually, by eye, to be centred on (936", 292'), deemed to be approximately the centre of the THEMIS FOV.

Notably the $\mathrm{He} \mathrm{I}_{3}$ line observed by THEMIS is optically thin, meaning that the bright tornado columns that we see are the integration of all points along the line of sight, with the majority of the emission coming from the prominence legs themselves. Since all the other observations showing the prominence in emission are in optically thick lines, it is necessary to find common features that can be used for co-alignment. As was noted previously and in Paper I, the prominence legs are visible in the Ca II images from SOT. We are therefore able to apply a threshold on an SOT image so that only the tornadoes are visible in emission, also blocking out the solar limb to replicate the appearance of the THEMIS image. In doing so, we are able to use get_correl_offsets to align the THEMIS data with the rest of the data sets. The offset for the THEMIS image is found to be $x=0.91^{\prime \prime}, y=0.61^{\prime \prime}$, meaning that the centre of the THEMIS map is $\left(936.91^{\prime \prime}, 292.61^{\prime \prime}\right)$.

\subsection{Hinode EIS with SDO AIA}

There is a known offset between the two EIS CCDs (Young et al. 2009; Graham et al. 2015), which is accounted for using the eis_ccd_offset routine. This routine ensures that the data at each wavelength is self-consistently spatially aligned. After each of the EIS maps at different wavelengths have been co-aligned, they must be aligned with the rest of the data sets. Since the emission in AIA images at $193 \AA$ is dominated by Fe XII, we use them alongside EIS Fe XII $195 \AA$ data for this co-alignment. Simple inspection of Fig. 5 shows that EIS and AIA images are very similar and have a good signal-to-noise ratio. We find that an offset of $x=4.08^{\prime \prime}$ and $y=18.24^{\prime \prime}$ needs to be applied to the EIS maps in order to bring them in line with the rest of the data.

The result of our co-alignment is shown in Fig. 6. This figure reveals how several structures observed with different instruments are related. The uncertainty on the spatial co-alignment of all data sets is estimated to be $2^{\prime \prime}$, the spatial resolution of the lowest resolution data used. Now that we have successfully co-aligned the data from THEMIS, AIA, EIS, SOT, and IRIS, we search for correlations on a pixel-by-pixel basis between the magnetic field and plasma parameters inferred from these observations. We first focus on THEMIS and IRIS data.

\section{Correlation between THEMIS and IRIS data}

We use Mg II line ratios and select some Mg II line parameters from Table 4 of Pereira et al. (2013) to study the properties of the plasma as seen by IRIS. To compare the plasma properties with the parameters of the magnetic field inferred from the THEMIS measurements, we first need to find the area that corresponds to the overlap between the IRIS raster and the THEMIS rasters. A mask of the area of overlap between IRIS and THEMIS is created, and with this mask we can find the appropriate pixels in both rasters to produce correlation plots on a pixel-by-pixel basis. However, as the spatial resolution of IRIS is higher in $y$ than that of THEMIS used here, we first average the IRIS data 


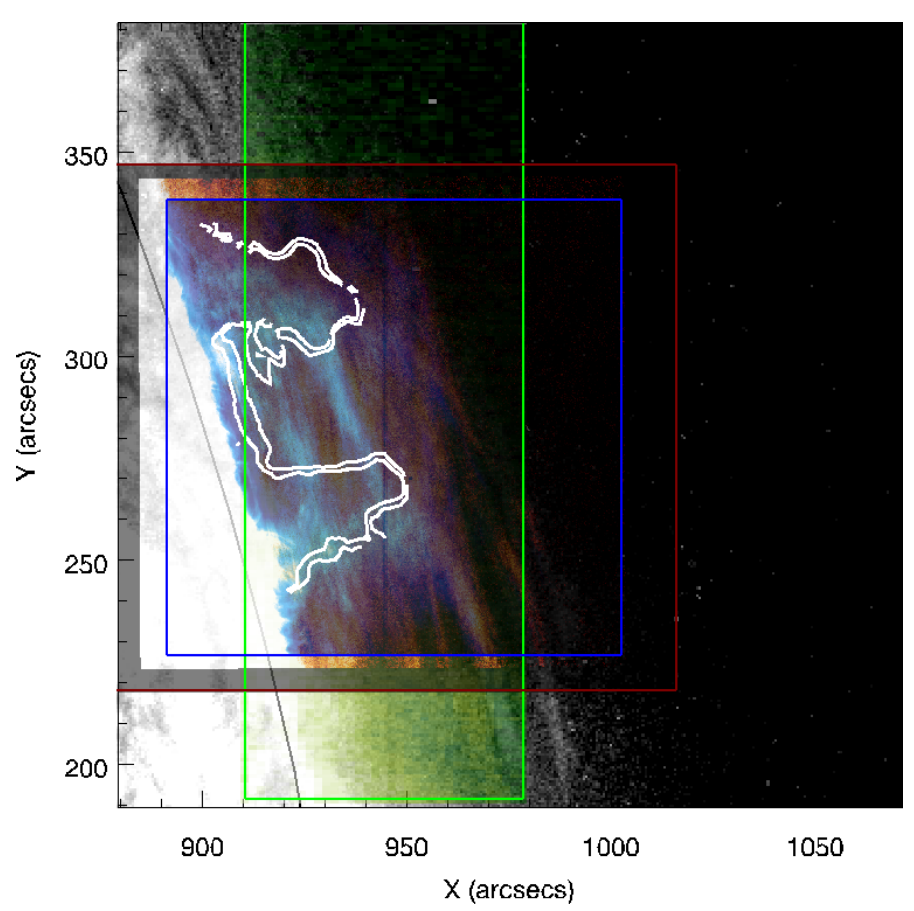

Fig. 6. Composite image of the prominence observed on 15 July 2014 showing, after co-alignment: the EIS raster in He II $256 \AA$ (green), the IRIS SJI in Mg II (red), and an SOT Ca II image (blue). The white contours show the THEMIS $\mathrm{D}_{3}$ intensity image. The background image is an AIA 304 Å image (greyscale).

over $1 "$ in $y$ to match the resolution of the THEMIS data. In the $x$-direction, IRIS has a $2^{\prime \prime}$ step, meaning that for each re-binned IRIS pixel there are two THEMIS magnetic field pixels. These are considered separately and are both compared to the same IRIS pixel.

Using the intensity map of the THEMIS raster, we can identify the locations of the tornadoes. This then allows us to create more masks for the pixels in the tornadoes and those outside them, in the rest of the prominence. The entirety of the overlapped area covered by the THEMIS raster is filled with prominence in the IRIS raster. These masks are shown in Fig. 7.

There is only a partial overlap between the two rasters, and therefore we only see the top of the northern tornado as seen by THEMIS and just over half of the southern one in the IRIS raster.

\section{1. $M g \| \mathrm{k}_{2} / \mathrm{k}_{3}$ ratio}

By measuring the level of reversal of the $\mathrm{k}$ line (equivalently for $\mathrm{h}$ ), which is defined as being the $\mathrm{Mg} \mathrm{II} \mathrm{k}_{2} / \mathrm{k}_{3}$ intensity ratio, we can compare these observations to models of the Mg II lines in prominences. Heinzel et al. (2014) gives us a grid of models with which to compare observations, allowing us to narrow down values for physical parameters such as gas pressure and temperature of the prominence. This analysis was done in Paper I for parts of this prominence, where it was found that the $\mathrm{k}_{2} / \mathrm{k}_{3}$ ratio was between 1 (non-reversed) and 2.8. Mg II $\mathrm{k}$ line profiles with a strong central reversal $\left(\mathrm{k}_{2} / \mathrm{k}_{3}>2\right)$ are reminiscent of those found in the chromosphere (Leenaarts et al. 2013; Pereira et al. 2013) and indicate that in these pixels the prominence plasma has a large optical thickness, probably due to high pressures (Heinzel et al. 2014).

Is the level of reversal related spatially to the magnetic field parameters? To investigate this question we have plotted the
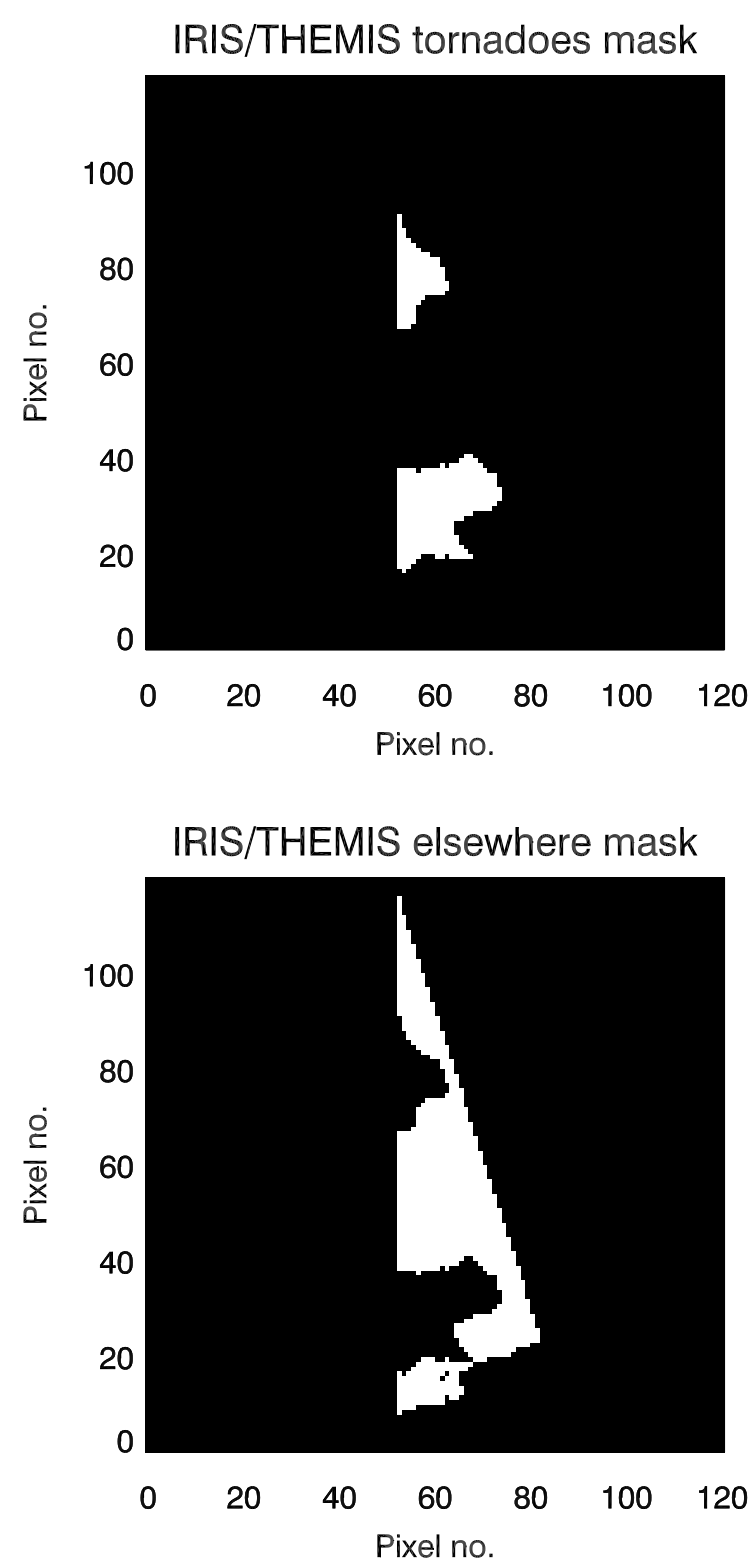

Fig. 7. Overlapped area between IRIS and THEMIS maps for data sets on 15 July 2014. White area shows overlap. Top: areas including the two tornadoes. Bottom: area including the rest of the prominence.

ratio $\mathrm{k}_{2} / \mathrm{k}_{3}$ in Fig. 8 against the magnetic field strength, inclination and azimuth. We present data separately for pixels defined as being "tornado" pixels, and "rest-of-prominence" pixels, using the masks described above. The value for the $k_{2} / k_{3}$ ratio for non-reversed profiles has been arbitrarily set to 0.5 in Fig. 8 to distinguish them. A black dashed line shows the cutoff value for reversed profiles.

The top panels of Fig. 8 show the $k_{2} / k_{3}$ ratio versus magnetic field strength in the tornadoes and the rest of the prominence. From these plots it appears that the field strength is generally higher in the tornadoes than the rest of the prominence, but there are no clear correlations between the field strength and the $\mathrm{k}_{2} / \mathrm{k}_{3}$ ratio. From the top panel of Fig. 9 it is clear that there is a higher field strength in the tornadoes than outside of them. In fact, points in the rest of the prominence have a mean field strength of $20 \mathrm{G}$, whereas in the tornadoes the majority of points have a value of around $30 \mathrm{G}$. 

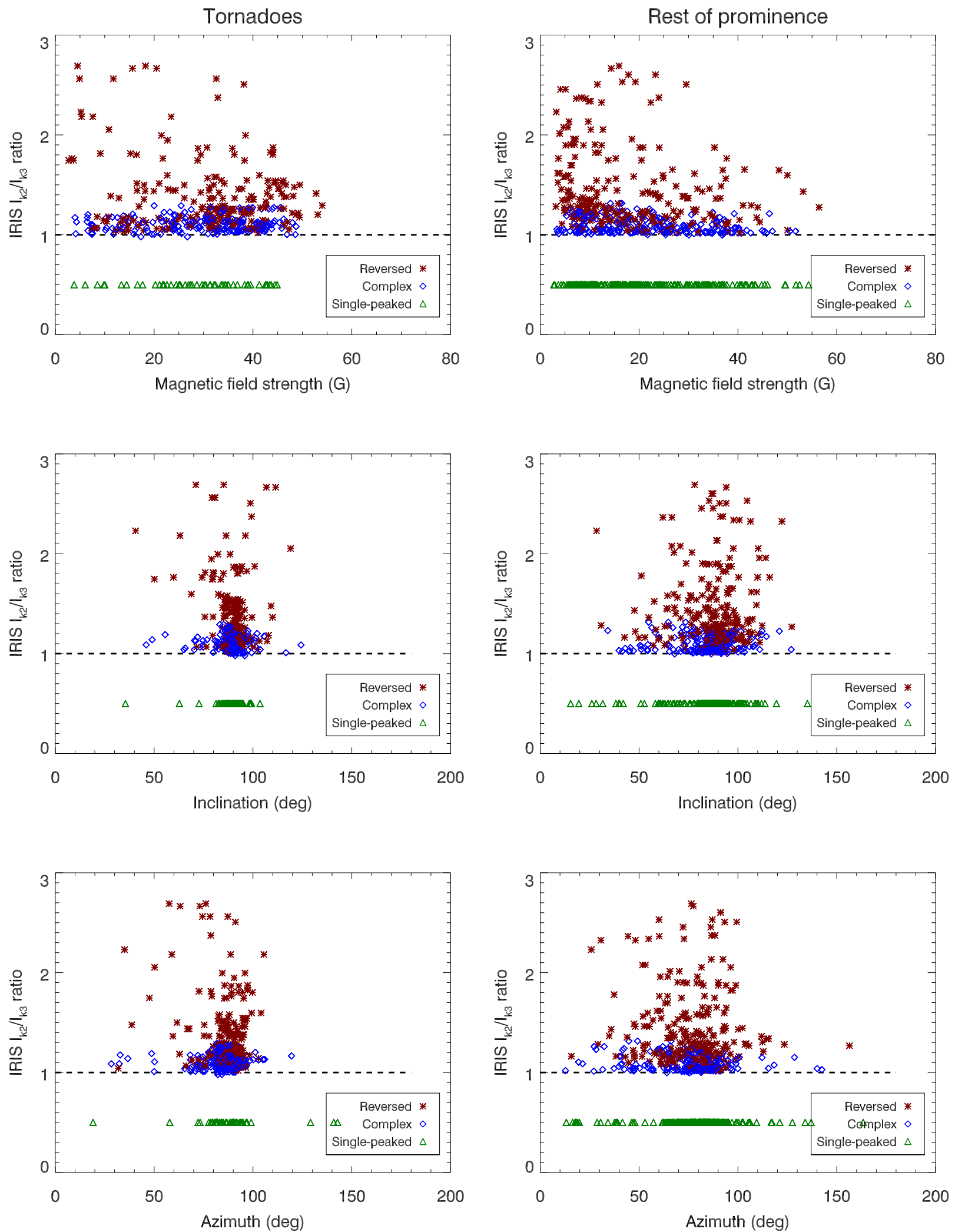

Fig. 8. $\mathrm{k}_{2} / \mathrm{k}_{3}$ ratio of $\mathrm{Mg}$ II against magnetic field parameters. Left column shows points in the tornadoes, right column shows points everywhere else in the prominence. Red asterisks are points where $\mathrm{Mg}$ II k profiles are reversed, green triangles are where Mg II k profiles are single peaked (manually placed at a ratio of 0.5 to distinguish them). Blue diamonds correspond to complex profiles, described in Sect. 2.1.2. The black dashed line provides a cutoff for reversed profiles, where a ratio of 1 corresponds to a single-peaked profile. Inclination is with respect to the local vertical and azimuth is with respect to the line of sight.

For the field inclination we see most of the points are clustered around an inclination of $90^{\circ}$ (i.e. horizontal) in Fig. 8 (middle panels). This is consistent with previous studies on this data
(Levens et al. 2016a,b), where it was found that the field is horizontal with respect to the limb. We see a few outliers in the tornadoes, but not a significant number. In the rest of the prominence 

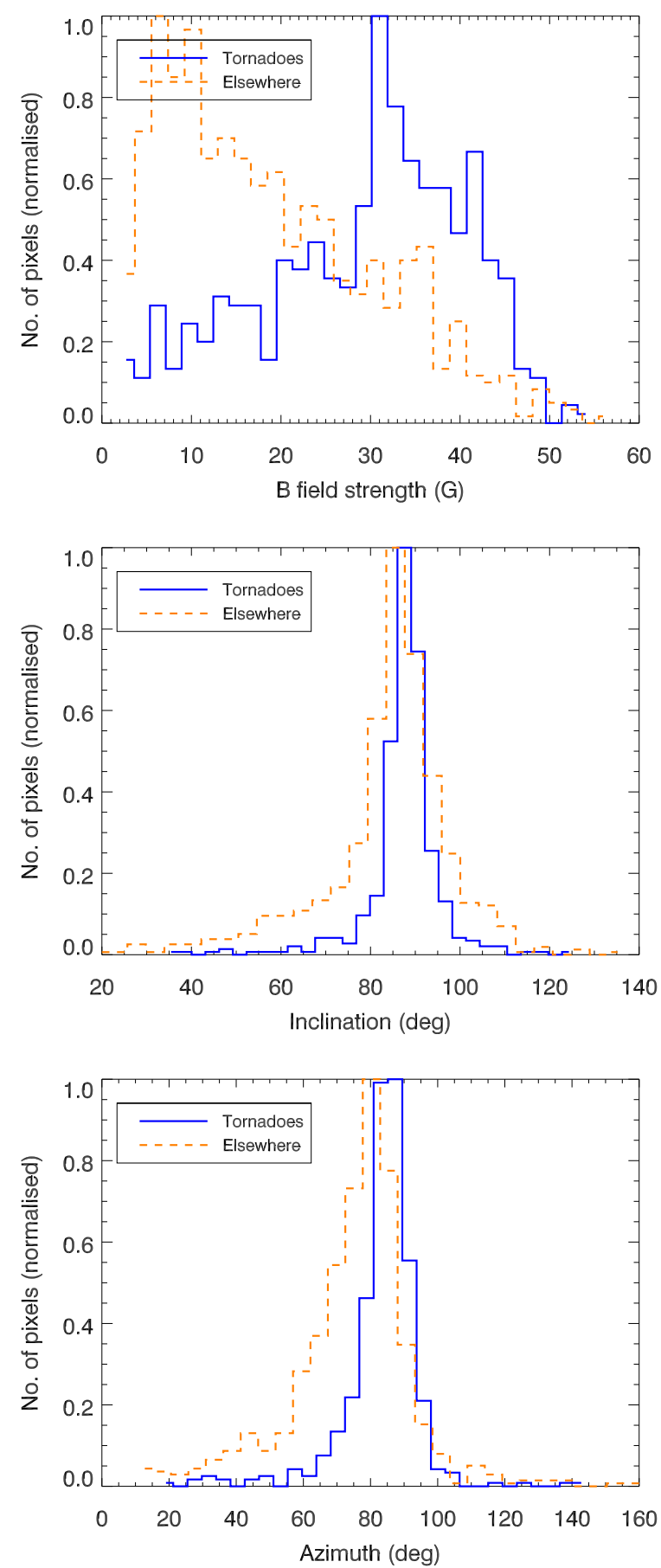

Fig. 9. Histograms of magnetic field parameters from THEMIS showing the distribution of values in both the tornadoes case ( $\sim 500$ pixels) and the rest-of-prominence case ( $\sim 1100$ pixels). Top: magnetic field strength. Middle: inclination. Bottom: azimuth.

there is a larger spread of values, but the field is still predominantly horizontal, as seen in Fig. 9 (middle panel).

A similar structure is seen in the $\mathrm{k}_{2} / \mathrm{k}_{3}$ ratio against azimuth (Fig. 8, bottom panels), where most of the points are clustered around a mean value of around $85^{\circ}$ in the tornadoes and $77^{\circ}$ elsewhere (Fig. 9, bottom panel). There is no clear correlation that can be seen.

We note that some of the points for the "complex" profiles have $k_{2} / k_{3}$ ratios lower than 1 in Fig. 8. This is due to the fact that the peaks given by the peak-finder algorithm (Waller et al., in prep.) are averaged over a number of pixels, but this averaging
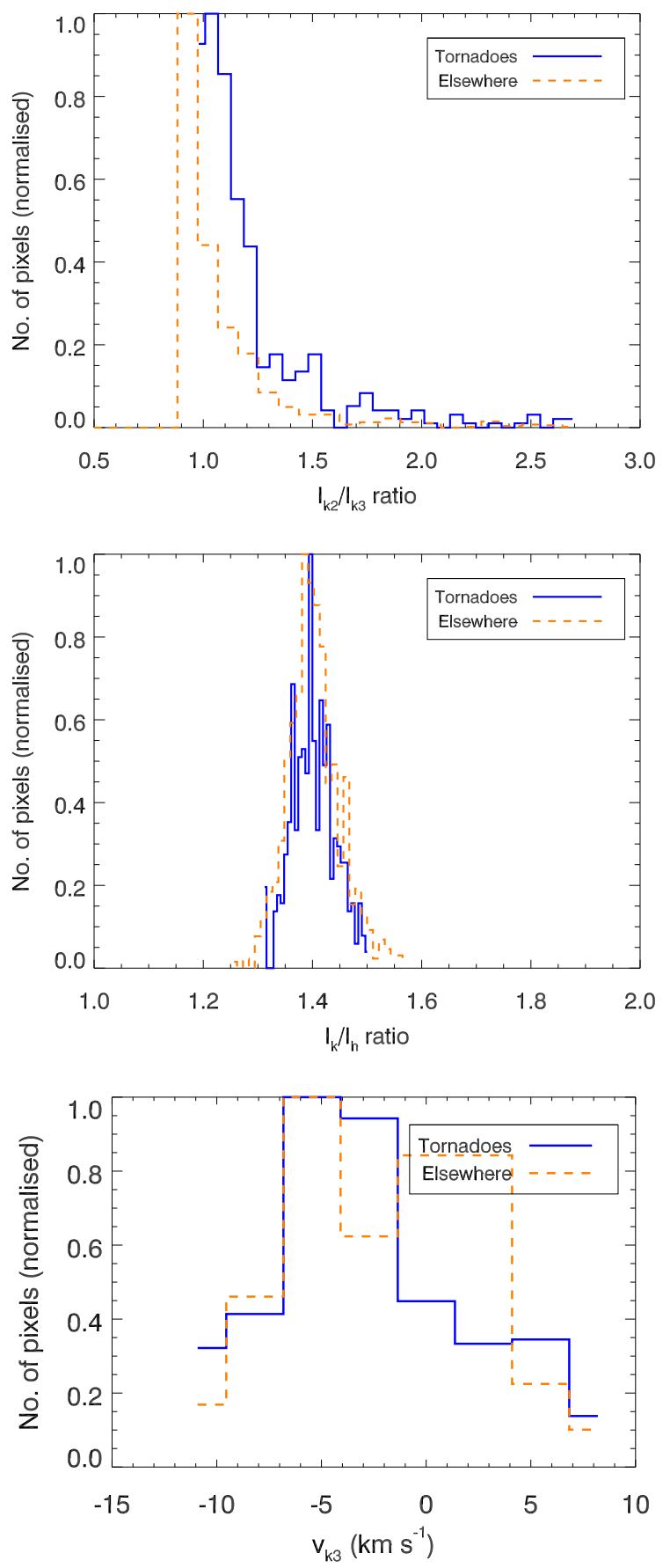

Fig. 10. Histograms of parameters derived from the $\mathrm{Mg}$ II lines showing the distribution of values in both the tornadoes case ( $\sim 500$ pixels $)$ and the rest-of-prominence case ( $\sim 1100$ pixels). Top: $\mathrm{k}_{2} / \mathrm{k}_{3}$ ratio (level of reversal). Middle: $\mathrm{k} / \mathrm{h}$ ratio. Bottom: $\mathrm{k}_{3}$ Doppler shift.

is not done for the intensity at the centroid of the distribution. Therefore in certain "complex" profiles where the peak is very sharp and narrow (i.e. one spectral pixel wide), the averaged peak intensity can be lower than the centroid intensity. As it is not a significant number of points and they are all "complex" profiles, we conclude that they are anomalies due to the automated handling of the profiles.

The top panel of Fig. 10 shows the distributions of the $\mathrm{k}_{2} / \mathrm{k}_{3}$ ratio in the tornadoes (solid blue) and elsewhere in the prominence (dashed orange). We take moments of the distribution, which tells us that the mean reversal level in the tornadoes 
is 1.23 , whereas in the rest of the prominence it is lower, with a mean value of 1.14. Standard deviation is 0.3 in both cases. There are also relatively more points extending to higher reversal levels in the tornadoes than elsewhere.

\section{2. $M g \| I_{k} / I_{h}$ ratio}

The relative intensities of the $\mathrm{h}$ and $\mathrm{k}$ lines of $\mathrm{Mg}$ II, referred to here as the $\mathrm{k} / \mathrm{h}$ ratio, can tell us something about the formation of these lines. Under normal chromospheric conditions, where the $\mathrm{Mg}$ II ions are collisionally excited, we would expect a $\mathrm{k} / \mathrm{h}$ ratio of 2 (Leenaarts et al. 2013) and the plasma to be optically thin. Previous prominence observations have shown typical $\mathrm{k} / \mathrm{h}$ ratios of around 1.5 (Schmieder et al. 2014; Liu et al. 2015; Vial et al. 2016; Levens et al. 2016a), and non local thermodynamic equilibrium (NLTE) models of Mg II have found similar expected values (Heinzel et al. 2014). Values lower than 2, as have been observed, suggest the presence of scattering in the emitting region, that is, a departure from the optically-thin case.

Figure 11 shows the $\mathrm{k} / \mathrm{h}$ ratio against the magnetic field parameters, with symbols and colours having the same meaning as in Fig. 8.

In the left-column panels (in the tornadoes) we see again that the data is more clustered towards higher field strengths than in the right-hand column, where there are many more points at lower field strength values. We find a similar behaviour to that seen in Fig. 8 in terms of inclination and azimuth. We find $\mathrm{k} / \mathrm{h}$ ratios similar to those reported previously of between 1 and 2 . We take moments of the $\mathrm{k} / \mathrm{h}$ ratio distributions (Fig. 10, middle panel) to find that in both the tornadoes and the rest of the prominence the ratio has a mean value of 1.41 , with standard deviation of 0.05 .

We also note that there are many more reversed and complex profiles in the tornado case than single peaked profiles. In the rest of the prominence we see relatively more single peaked profiles.

\section{3. $M g \| k_{3}$ Doppler shift}

The $\mathrm{k}_{3}$ feature at the $\mathrm{Mg}$ II $\mathrm{k}$ line centre corresponds to the most optically-thick part of that line. When we observe features of a prominence at wavelengths near line centre, we are really only looking at emission from the frontmost layer(s) of material. Emission from material behind that is almost all re-absorbed or scattered out of the line of sight at these wavelengths. By then measuring the deviation of this $\mathrm{k}_{3}$ feature of reversed profiles from rest wavelength, we can theoretically measure the velocity of the parts of the prominence emitting in $\mathrm{Mg}$ II that are closest to us, the observer. Does an increase in magnetic field strength, and hence magnetic pressure, correspond to higher plasma velocities? This is what could be expected in a low- $\beta$ plasma - observed motions in a low- $\beta$ plasma will be caused by the magnetic pressure.

Figure 12 shows plots of $\mathrm{k}_{3}$ Doppler shift versus the magnetic field parameters, the same as seen in Figs. 8 and 11. The points are at discrete values of velocity due to the spectral resolution of IRIS. The position of the $\mathrm{k}_{3}$ minimum cannot be measured more accurately without fitting the curve with a Gaussian, for example. However, there is no reason to assume a Gaussian profile for an optically-thick line. We also note that the actual Doppler shifts encapsulated in each discrete point cover a range of $v_{k 3}$ values. This is conveyed in the bottom panel of Fig. 10, where bin sizes reflect the spectral resolution of IRIS.
In the case of the $\mathrm{k}_{3}$ Doppler shift versus magnetic field strength in the tornado (top left panel of Fig. 12), we see that higher line of sight velocities are found at higher magnetic field strengths, and at lower magnetic field strengths the $\mathrm{k}_{3}$ Doppler shift is much closer to zero. However, in the rest of prominence case, the opposite appears to be true. This could be an indication that the plasma $\beta$ is lower in the tornado than in the rest of the prominence. In all cases these velocities are within around $\pm 10 \mathrm{~km} \mathrm{~s}^{-1}$, which is a similar value to that found previously in quiescent prominences with IRIS (Paper I). Comparing $\mathrm{k}_{3}$ Doppler shift to inclination and azimuth of the magnetic field, we see again the inclination clustered around $90^{\circ}$, and azimuth values of around $80-90^{\circ}$ in the tornado points.

\section{Correlation between THEMIS and EIS data}

In order to identify correlations between the profiles observed by EIS and the magnetic field parameters from THEMIS, we first need to characterise the spectral lines seen by EIS. To do this we follow the outline laid out by Young et al. (2007), and fit Gaussian profiles to the spectral lines. As noted in that paper and elsewhere, complex blended lines are found in EIS data, many of which can be seen in prominence observations (see Labrosse et al. 2011; Levens et al. 2015, for more details). We here follow a similar strategy to Levens et al. (2015) in the deblending of these lines where necessary.

In the EIS study used here, spectral lines formed at a range of plasma temperatures are available - from He II $256 \AA$, formed at $\sim 30000 \mathrm{~K}$, up to Fe XV at $283 \AA$, formed at $\sim 2.5 \mathrm{MK}$. Unfortunately the lower temperature lines available are mostly either part of a large blend with hotter components (such as He II and the O v lines at $192 \AA$ ), or suffer from poor signal-to-noise ratios (such as the O VI lines around $184 \AA$ or the Si VII lines at 274 and $275 \AA$ ).

We ideally want to study two lines formed by the same ion whose intensity ratio shows some density sensitivity, a process outlined in Young et al. (2007). For this analysis we chose the two Fe XII lines at $195.119 \AA$ (the strongest line as observed by EIS) and $195.179 \AA$, which are de-blended by fitting two Gaussian profiles whose centroid positions are tied at a fixed distance apart. These are the same lines used by Levens et al. (2015) on another tornado-like prominence. Figure 5 shows the two intensity maps for these lines. The prominence can be seen as two dark columns, with the northern column being much more visible than the southern one, as is the case in coronal AIA images (Figs. 2, 5).

Fe XII is formed at a plasma temperature of $1.5 \times 10^{6} \mathrm{~K}$, much higher than the temperatures expected in a prominence. However, as previous analysis has shown (Levens et al. 2015), it appears that the tornado structure can be traced to temperatures as high as this through a prominence-corona transition region (PCTR), with hot plasma seemingly forming a "sheath" around the cool core. In fact, the core of the prominence is formed from many threads and sheaths may exist along each thread, as was suggested in the multi-threaded model of prominence formation (Luna et al. 2012).

These two iron lines show density sensitivity across a range of densities, so are suitable for this analysis. Using atomic data from CHIANTI v8.0 (Dere et al. 1997; Del Zanna et al. 2015), we can create a density map at the formation temperature of these two lines $\left(1.5 \times 10^{6} \mathrm{~K}\right)$. The result of this is shown in Fig. 13. 

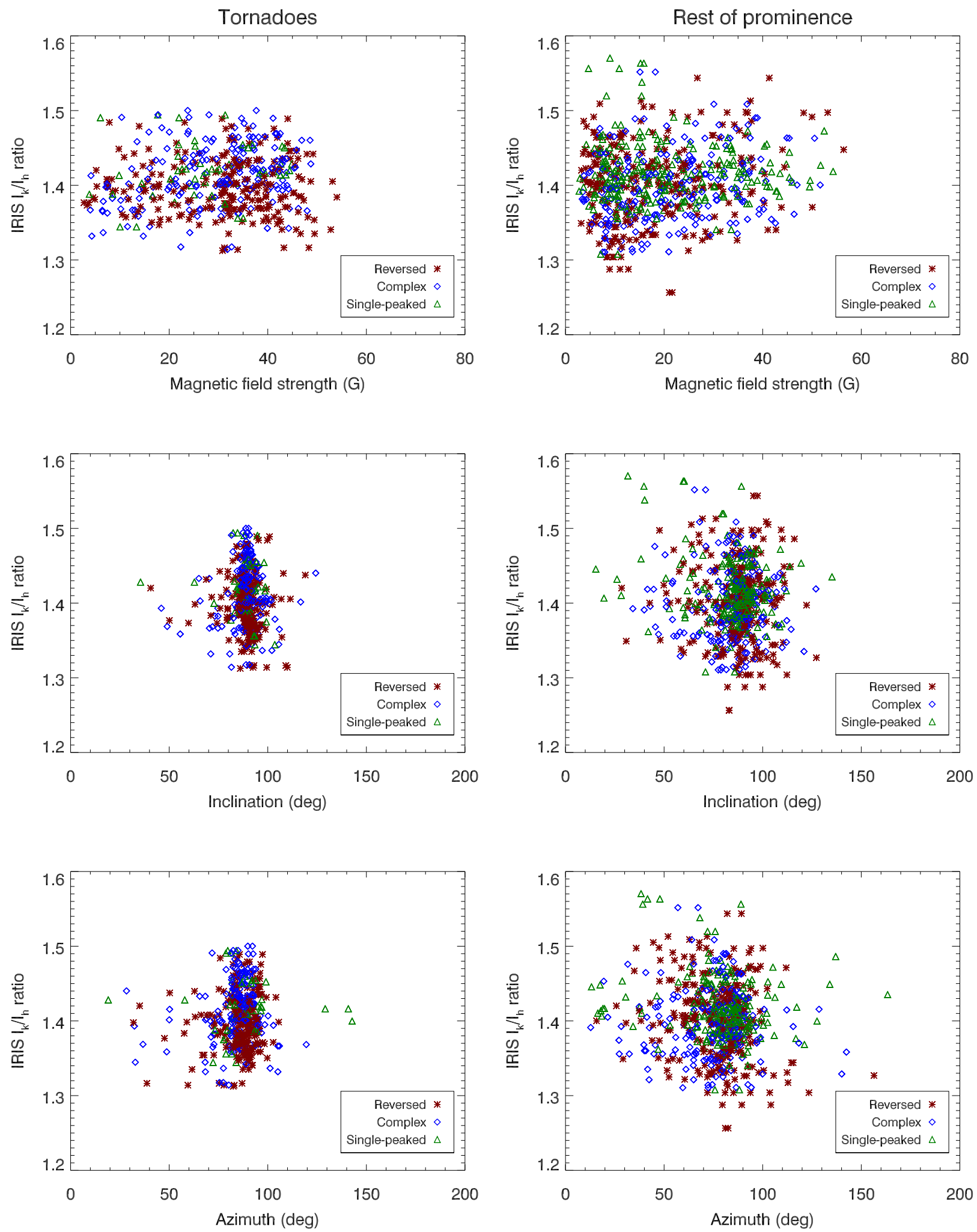

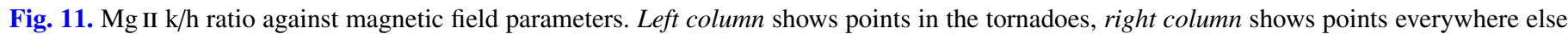
in the prominence. Red asterisks are points where $\mathrm{Mg}$ II k line profiles are reversed, green triangles are where $\mathrm{Mg}$ II $\mathrm{k}$ profiles are single peaked. Blue diamonds correspond to complex profiles, described in Sect. 2.1.2. Inclination is with respect to the local vertical, and azimuth is with respect to the line of sight.

We then need to calculate the overlapping region between the EIS raster and the THEMIS raster, as was done with IRIS in Sect. 4. The overlap masks for EIS and THEMIS are shown in Fig. 14. Notably for this correlation we only consider "tornado" points to be those in the northern tornado. This is done because the southern tornado is barely visible in the Fe XII lines considered here, mostly blocked by bright coronal emission in front of it. Following this, we can perform a statistical analysis 

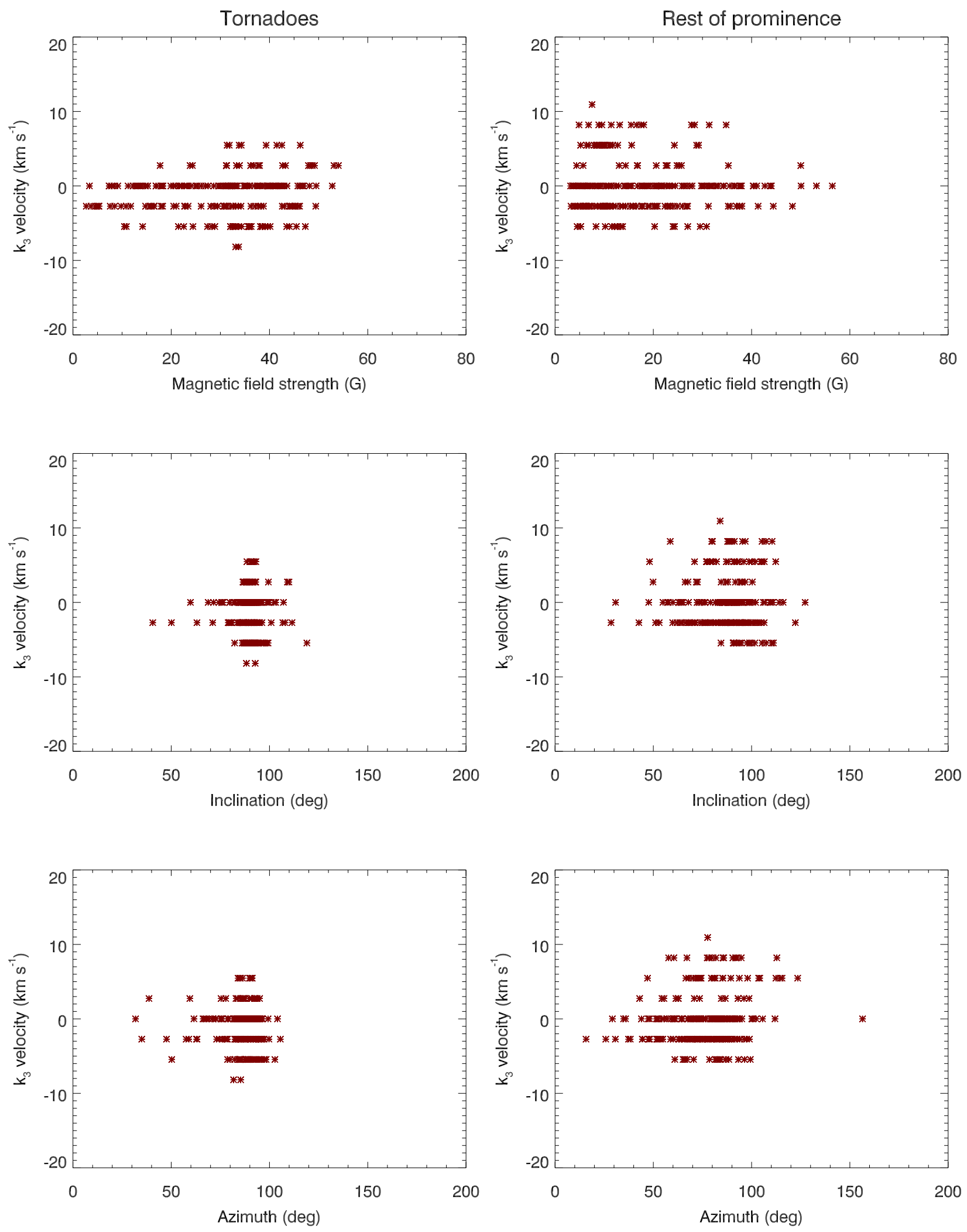

Fig. 12. $\mathrm{Mg}_{\text {II }} \mathrm{k}_{3}$ Doppler shift, relative to the nominal rest wavelength of $\mathrm{Mg}$ II, against magnetic field parameters. Left column shows points in the tornadoes, right column shows points everywhere else in the prominence. Points are all taken from pixels where Mg II profiles are reversed. Inclination is with respect to the local vertical, and azimuth is with respect to the line of sight.

between points in the EIS and THEMIS rasters. Figure 15 shows the scatter plots of electron density versus magnetic field parameters from THEMIS. The panels in this plot are in the same order as those in Figs. 8, 11, and 12. We see in these plots a relatively small scatter in densities, with most points having $\log n_{\mathrm{e}}$ between 8.5 and 9.5. The mean electron density at $T \sim 1.5 \times 10^{6} \mathrm{~K}$ is 

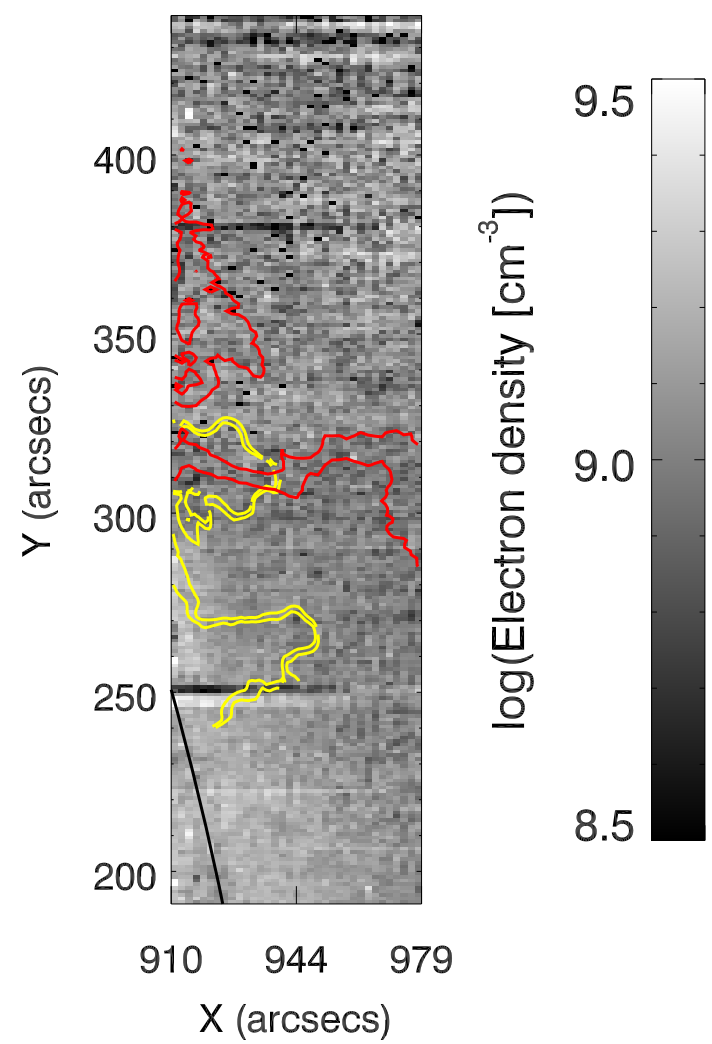

Fig. 13. Density map of the prominence region using the density sensitive Fe XII lines at 195.119 ̊ and 195.179 $\AA$. Yellow contours show outlines of the tornadoes as seen by THEMIS. Red contours are $22 \%$ and $25 \%$ of the EIS $195.119 \AA$ intensity, the same as the white contours in Fig. 5. Black line shows the solar limb position.

lower in the tornado than in the surrounding corona, as is clear by looking at the histogram of Fig. 16. In the tornado the mean density is $\log n_{\mathrm{e}}=8.98 \pm 0.14$, whereas in the surrounding corona the value is $\log n_{\mathrm{e}}=9.06 \pm 0.14$, with $n_{\mathrm{e}}$ in units of $\mathrm{cm}^{-3}$. This is comparable with the results of Levens et al. (2015). The lower density in the tornado could be due to the volume blocking effect of cool material in the tornado region (Heinzel et al. 2008); cool material in the tornado means that there is less hot Fe XII plasma along that line of sight.

\section{Correlation between IRIS and EIS data}

We also look for correlations between the Mg II line ratios and plasma parameters and the electron densities from EIS. Figure 17 shows plots of electron density versus (from top to bottom) $\mathrm{Mg} \mathrm{II} \mathrm{k}_{2} / \mathrm{k}_{3}$ ratio, $\mathrm{k} / \mathrm{h}$ ratio, and $\mathrm{k}_{3}$ Doppler shift. As can be seen in Fig. 2, the IRIS raster lies entirely inside the EIS raster, so the overlap area is the IRIS raster field of view. The EIS spatial resolution is lower than that of IRIS, so the IRIS data is binned spatially to match the EIS resolution, as described in Sect. 4 for comparing IRIS and THEMIS data.

The points are again divided into those in the northern tornado and those outside of it. This is done using the THEMIS brightness, although we note that this region misses out the highest altitude parts of the tornado. We only consider the northern tornado here, as in Sect. 5.

The plots in Fig. 17 show that there are no meaningful correlations between the IRIS line ratios or plasma parameters and the electron density. We note that the plasma emitting
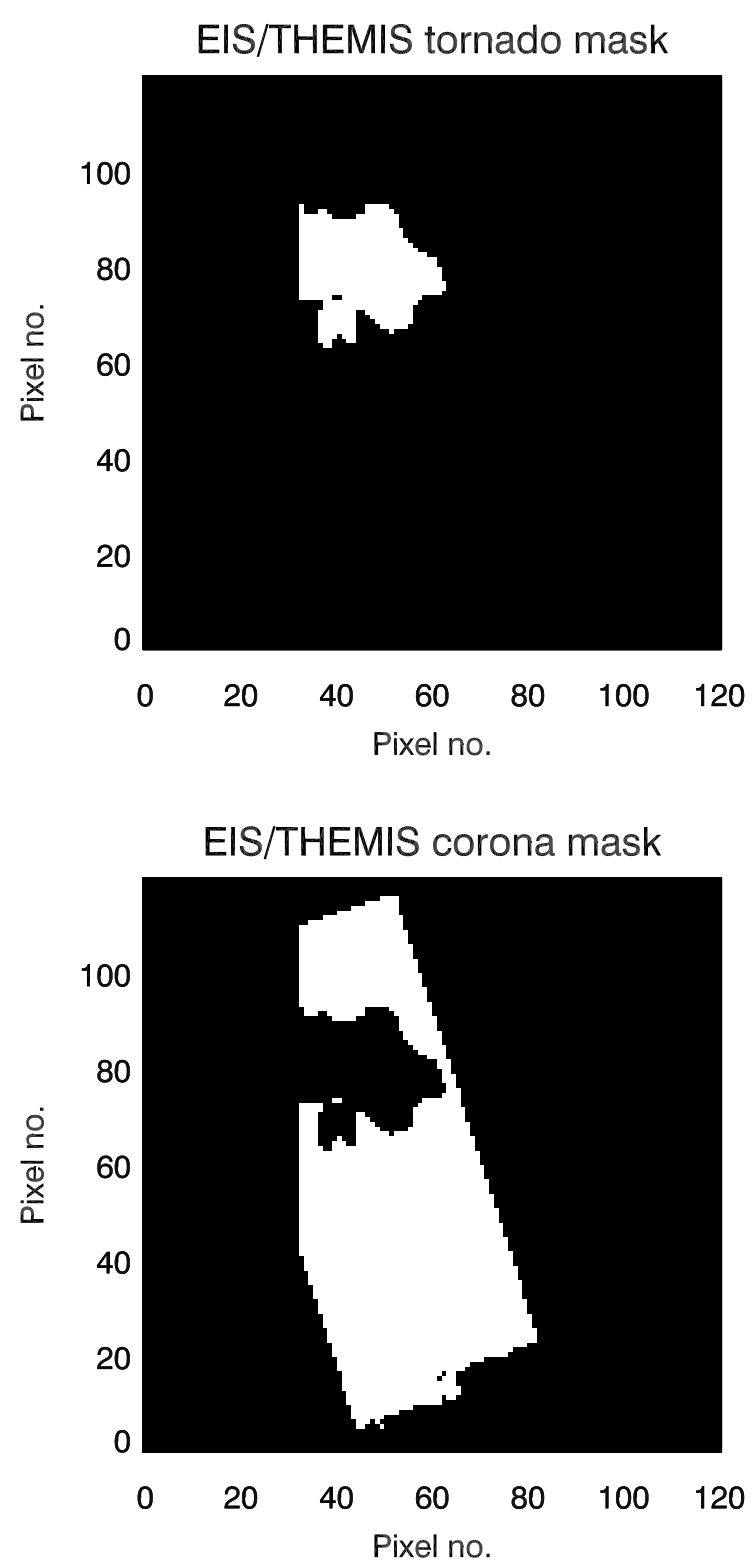

Fig. 14. Overlapped area between EIS and THEMIS maps for data sets on 15 July 2014. White area shows overlap. Top: area including the northern tornado, which is visible in the EIS $195 \AA$ line. Bottom: area including the surrounding corona.

the lines observed by the two instruments is formed under very different circumstances: the Fe XII emission seen by EIS is formed at coronal temperatures, and is optically thin, whereas the $\mathrm{Mg}$ II $\mathrm{k}$ line that IRIS observes is formed at chromospheric temperatures, and is optically thick. This could explain why these correlation plots do not show any patterns.

\section{Conclusion}

In this paper we try to ascertain whether there are correlations between magnetic field parameters, calculated from observations from a ground-based spectropolarimeter, and plasma parameters derived from UV and EUV spectroscopic observations from space, using data from the 15 July 2014 obtained during a joint observing campaign with the satellites IRIS and Hinode, and the telescope THEMIS in the Canary Islands. This prominence 

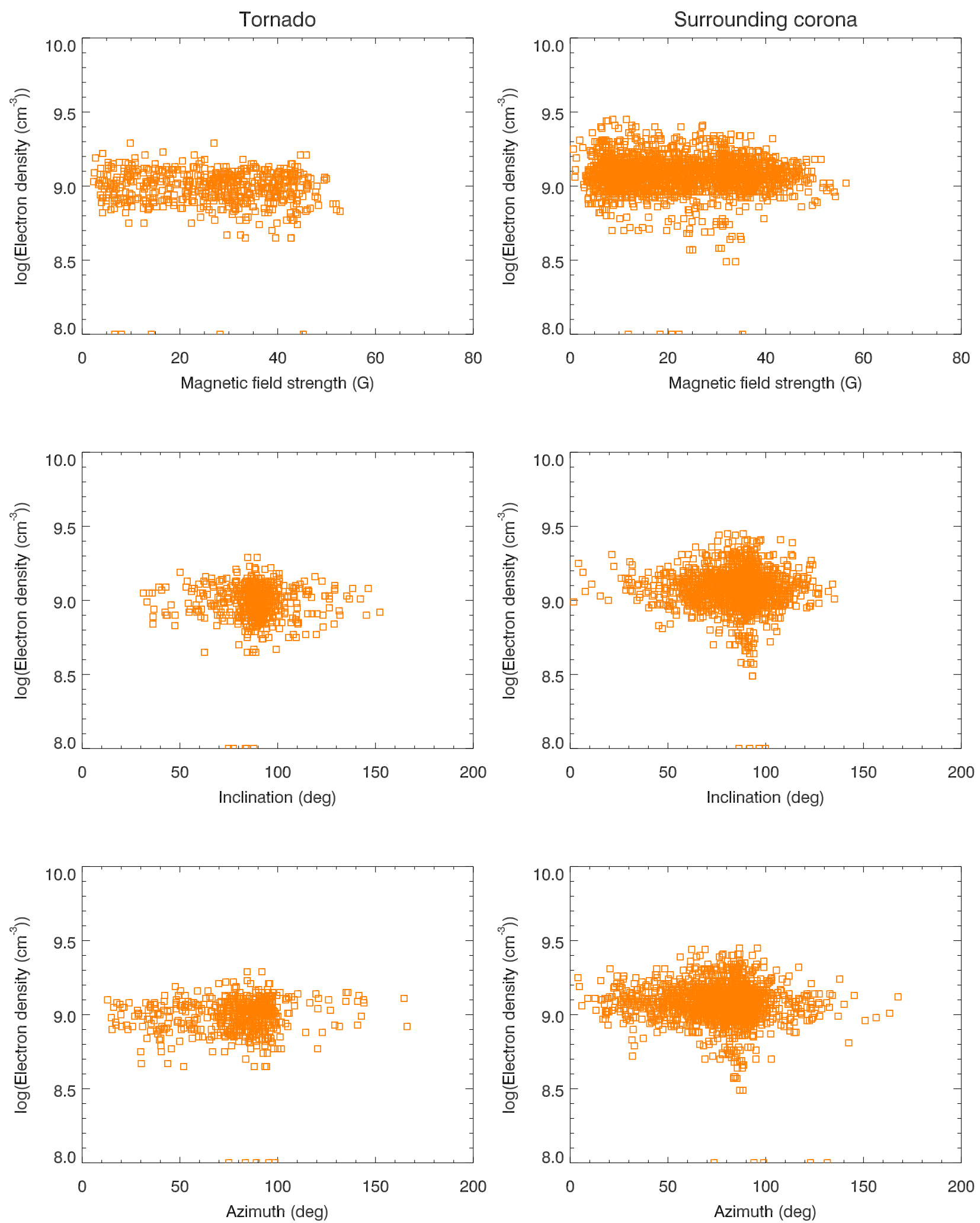

Fig. 15. Electron density, calculated using the intensity ratio of the Fe XII lines 195.119/195.179 observed by EIS, against magnetic field parameters. Left column shows points in the tornadoes, right column shows points in the surrounding corona.

has also been studied in Levens et al. (2016a). This data set was chosen as it shows fairly good coverage of the prominence of interest, with data available from each of our instruments with relatively good spatial overlap. THEMIS observations, however, were obtained a few hours after the satellite observations.
The first challenge is the co-alignment of data sets from different instruments. We use a $2 \mathrm{D}$ cross-correlation method on similar images from each instrument to co-align the data, allowing us to compare the data on a pixel-by-pixel basis. From this study we conclude that there are no correlations between the 


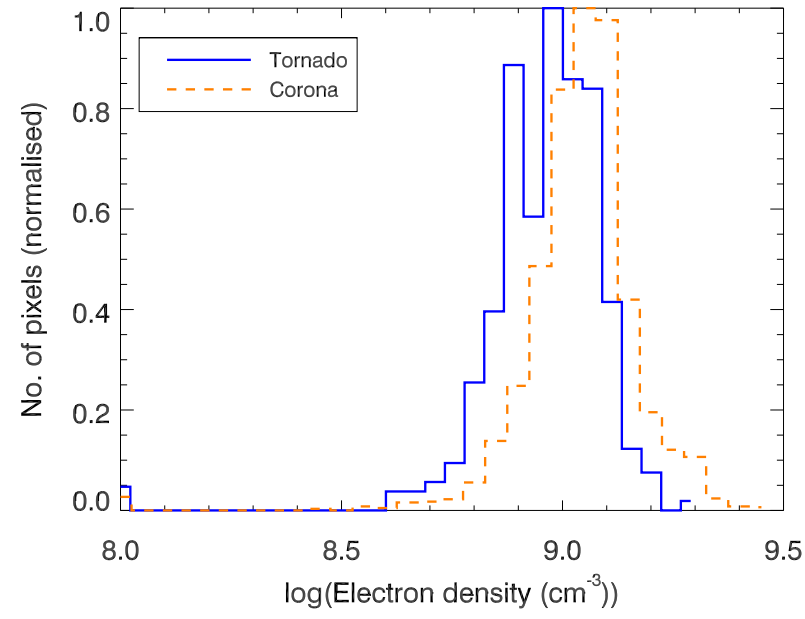

Fig. 16. Histograms of electron density showing the distribution of values in both the northern tornado ( $\sim 600$ pixels) and the surrounding corona ( 3000 pixels).

magnetic field parameters from THEMIS and the Mg II parameters from the IRIS observation, nor between the magnetic field parameters and electron densities calculated from Hinode EIS. We find that the magnetic field is generally stronger within the tornadoes $(\sim 30 \mathrm{G})$ than outside them $(\sim 20 \mathrm{G})$, and that the inclination and azimuth are the same as values found previously (Levens et al. 2016b).

We study the level of reversal in the $\mathrm{Mg}$ II $\mathrm{h}$ and $\mathrm{k}$ lines, and find that it varies from unreversed to a $\mathrm{k}_{2} / \mathrm{k}_{3}$ ratio of around 2.8 , suggesting high optical thickness at these locations. The mean reversal level is found to be 1.23 in the tornadoes and 1.14 in the rest of the prominence, suggesting that there are relatively more reversed profiles in the tornadoes than elsewhere. We see a small spread of $\mathrm{k} / \mathrm{h}$ ratios, ranging from around 1.3 to 1.5 . For optically-thin, collisionally-excited emission we would expect a $\mathrm{k} / \mathrm{h}$ ratio of 2 (Leenaarts et al. 2013), with departures from this value indicating a departure from the optically-thin regime. The mean $\mathrm{k} / \mathrm{h}$ ratio takes a value of 1.41 in both the tornadoes and in the rest of the prominence. This is a similar value as found previously (Schmieder et al. 2014; Liu et al. 2015; Levens et al. 2016a; Vial et al. 2016). The displacement of the $\mathrm{k}_{3}$ feature from its stationary position at line centre gives an indication of how the frontmost layers of the prominence are moving along the line of sight. We measure Doppler shifts of the $\mathrm{k}_{3}$ reversal between around $\pm 10 \mathrm{~km} \mathrm{~s}^{-1}$ everywhere. This is comparable to the overall velocity distribution, outlined in Paper I. For a quiescent prominence, we do not expect the overall velocity to be high, and it follows in that case that the $\mathrm{k}_{3}$ Doppler shift would also be low.

We also present a comparison of the electron density at a temperature of $1.5 \times 10^{6} \mathrm{~K}$, as calculated from EIS Fe XII observations, with the magnetic field parameters from THEMIS. The electron densities used here were estimated by calculating the intensity ratio of the Fe XII 195.119 ̊ and 195.179 ̊ lines, and then comparing this to the density curve for that ratio as computed by CHIANTI. We find a small scatter of densities in the prominence, with electron densities generally between $\log n_{\mathrm{e}}$ between 8.5 and 9.5, with a slightly larger scatter in the tornadoes pixels. The mean electron density is higher in the corona than in the tornado considered here, with values of $\log n_{\mathrm{e}}=9.06 \pm 0.14$ and $\log n_{\mathrm{e}}=8.98 \pm 0.14$ respectively. This is comparable to previous studies on the electron density in tornado-like prominences (Levens et al. 2015). The lower electron density along the line of
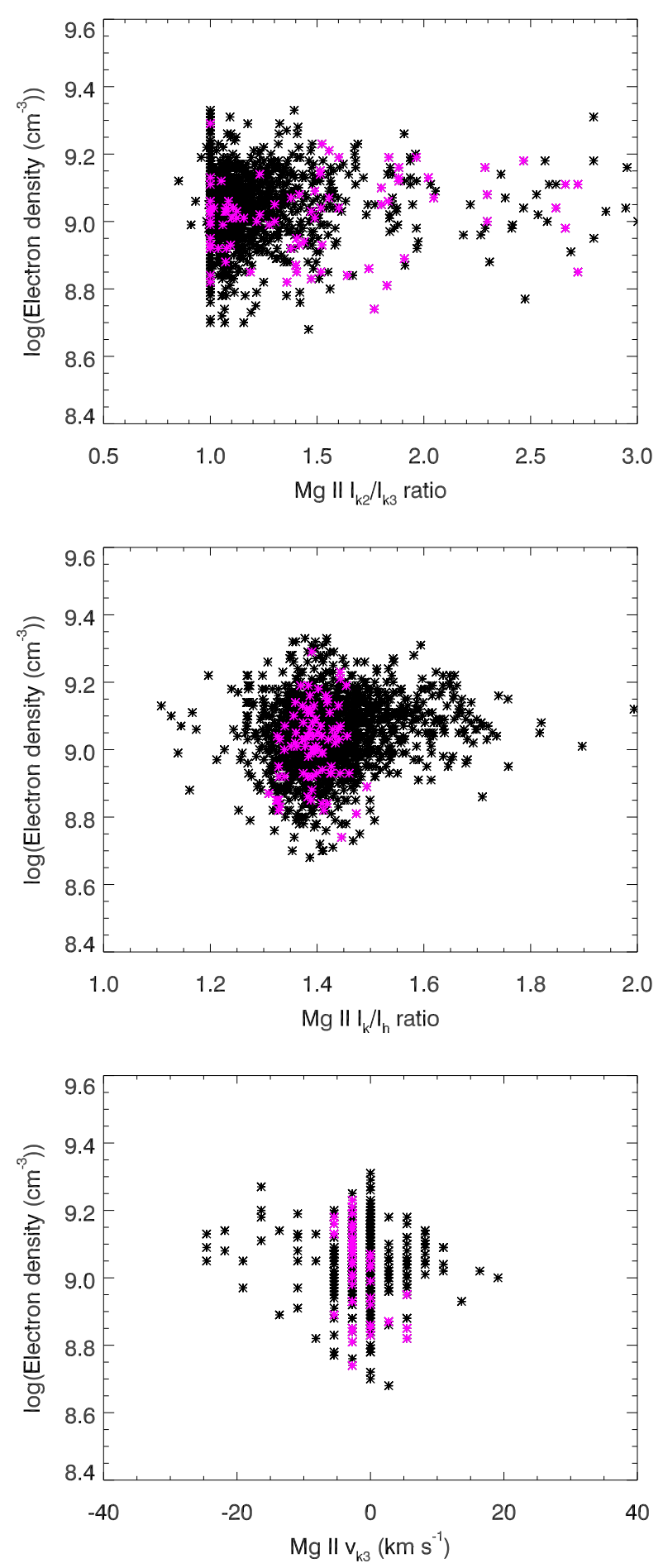

Fig. 17. Correlation plots showing electron density, calculated using the intensity ratio of the Fe XII lines 195.119/195.179 observed by EIS, against: top: $\mathrm{Mg} \mathrm{II} \mathrm{k}_{2} / \mathrm{k}_{3}$ ratio. Middle: $\mathrm{Mg}$ II k/h ratio. Bottom: $\mathrm{Mg}_{\text {II }} \mathrm{k}_{3}$ Doppler shift, from IRIS. Magenta points show points in the northern tornado in both rasters. Black points are for the rest of the overlapped region.

sight of the tornado could be due to volume blocking by the cool material at the tornado location (Heinzel et al. 2008). No correlation is found between the electron density and the line and plasma parameters derived from the $\mathrm{Mg}$ II lines.

While no clear correlation is found between line parameters and magnetic field parameters, we provide the first detailed maps 
of such parameters for a prominence including a tornado. It is important to note that the observations are not strictly simultaneous. We also note that the overlap between the data sets does not fully cover the region of interest, so we do not have a complete picture of the correlation at all parts of the prominence, especially in the IRIS versus THEMIS plots. More importantly, there are differences in the optical thickness of the lines used in

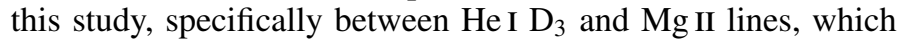
could be having an adverse effect on potential correlations as the magnetic field information retrieved from the inversion of the $\mathrm{D}_{3}$ spectro-polarimetric data represents averaged quantities along the line of sight, whereas the IRIS observations allow us to see mostly the frontmost part of the tornado and surrounding prominence. In this respect, similar studies in the future should make use of diagnostics based on spectral lines with comparable optical thicknesses. The recent observations by the Chromospheric Lyman-Alpha SpectroPolarimeter (CLASP; Kano et al. 2012; Kobayashi et al. 2012) and the available spectropolarimetric measurements in the optically-thick hydrogen Lyman $\alpha$ line may help to measure plasma and magnetic field parameters in similar regions of the prominence.

Acknowledgements. The authors thank S. Gunar, B. Gelly and the team at the THEMIS telescope for assisting with these observations. P.J.L. acknowledges support from an STFC Research Studentship ST/K502005/1. N.L. acknowledges support from STFC grant ST/L000741/1. We are grateful for the financial support from the International Space Science Institute where this work was presented, and thank our colleagues from International Team 374 for useful and insightful discussions. The AIA data are provided courtesy of NASA/SDO and the AIA science team. IRIS is a NASA small explorer mission developed and operated by LMSAL with mission operations executed at the NASA Ames Research Center and major contributions to downlink communications funded by the Norwegian Space Center (NSC, Norway) through an ESA PRODEX contract. Hinode is a Japanese mission developed and launched by ISAS/JAXA, with NAOJ as domestic partner and NASA and STFC (UK) as international partners.

\section{References}

Aulanier, G., \& Démoulin, P. 1998, A\&A, 329, 1125

Casini, R., López Ariste, A., Tomczyk, S., \& Lites, B. W. 2003, ApJ, 598, L67

Culhane, J. L., Harra, L. K., James, A. M., et al. 2007, Sol. Phys., 243, 19

De Pontieu, B., Title, A. M., Lemen, J. R., et al. 2014, Sol. Phys., 289, 2733

Del Zanna, G., Dere, K. P., Young, P. R., Landi, E., \& Mason, H. E. 2015, A\&A, 582, A56

Dere, K. P., Landi, E., Mason, H. E., Monsignori Fossi, B. C., \& Young, P. R. 1997, A\&AS, 125, 149

Graham, D. R., Fletcher, L., \& Labrosse, N. 2015, A\&A, 584, A6
Gunár, S., Heinzel, P., Anzer, U., \& Schmieder, B. 2008, A\&A, 490, 307 Heinzel, P., Schmieder, B., Fárník, F., et al. 2008, ApJ, 686, 1383 Heinzel, P., Vial, J.-C., \& Anzer, U. 2014, A\&A, 564, A132 Heinzel, P., Schmieder, B., Mein, N., \& Gunár, S. 2015, ApJ, 800, L13

Kano, R., Bando, T., Narukage, N., et al. 2012, in Space Telescopes and Instrumentation 2012: Ultraviolet to Gamma Ray, Proc. SPIE, 8443, 84434

Kobayashi, K., Kano, R., Trujillo-Bueno, J., et al. 2012, in Fifth Hinode Science Meeting, eds. L. Golub, I. De Moortel, \& T. Shimizu, ASP Conf. Ser., 456, 233

Kosugi, T., Matsuzaki, K., Sakao, T., et al. 2007, Sol. Phys., 243, 3

Labrosse, N., \& Gouttebroze, P. 2001, A\&A, 380, 323

Labrosse, N., \& Rodger, A. S. 2016, A\&A, 587, A113

Labrosse, N., Schmieder, B., Heinzel, P., \& Watanabe, T. 2011, A\&A, 531, A69

Leenaarts, J., Pereira, T. M. D., Carlsson, M., Uitenbroek, H., \& De Pontieu, B. 2013, ApJ, 772, 89

Lemen, J. R., Title, A. M., Akin, D. J., et al. 2012, Sol. Phys., 275, 17

Levens, P. J., Labrosse, N., Fletcher, L., \& Schmieder, B. 2015, A\&A, 582, A27

Levens, P. J., Schmieder, B., Labrosse, N., \& López Ariste, A. 2016a, ApJ, 818, 31

Levens, P. J., Schmieder, B., López Ariste, A., et al. 2016b, ApJ, 826, 164

Li, X., Morgan, H., Leonard, D., \& Jeska, L. 2012, ApJ, 752, L22

Liu, W., De Pontieu, B., Vial, J.-C., et al. 2015, ApJ, 803, 85

López Ariste, A., \& Casini, R. 2002, ApJ, 575, 529

López Ariste, A., Rayrole, J., \& Semel, M. 2000, A\&AS, 142, 137

López Ariste, A., Asensio Ramos, A., Manso Sainz, R., Derouich, M., \& Gelly, B. 2009, A\&A, 501, 729

Luna, M., Karpen, J. T., \& DeVore, C. R. 2012, ApJ, 746, 30

Luna, M., Moreno-Insertis, F., \& Priest, E. 2015, ApJ, 808, L23

Orozco Suárez, D., Asensio Ramos, A., \& Trujillo Bueno, J. 2014, A\&A, 566, A46

Martinez Gonzalez, M. J., Asensio Ramos, A., Arregui, I., et al. 2016, ApJ, 825, 119

Panasenco, O., Martin, S. F., \& Velli, M. 2014, Sol. Phys., 289, 603

Panesar, N. K., Innes, D. E., Tiwari, S. K., \& Low, B. C. 2013, A+A, 549, A105 Pereira, T. M. D., Leenaarts, J., De Pontieu, B., Carlsson, M., \& Uitenbroek, H. 2013, ApJ, 778, 143

Schmieder, B., Tian, H., Kucera, T., et al. 2014, A\&A, 569, A85

Schmieder, B., Zapior, M., López Ariste, A., et al. 2017a, A\&A, 606, A30

Schmieder, B., Mein, P., Mein, N., et al. 2017b, A\&A, 597, A109

Su, Y., Wang, T., Veronig, A., Temmer, M., \& Gan, W. 2012, ApJ, 756, L41

Su, Y., Gömöry, P., Veronig, A., et al. 2014, ApJ, 785, L2

Suematsu, Y., Tsuneta, S., Ichimoto, K., et al. 2008, Sol. Phys., 249, 197

Tsuneta, S., Ichimoto, K., Katsukawa, Y., et al. 2008, Sol. Phys., 249, 167

Vial, J. C. 1982, ApJ, 253, 330

Vial, J.-C., Pelouze, G., Heinzel, P., Kleint, L., \& Anzer, U. 2016, Sol. Phys., 291, 67

Wedemeyer, S., Scullion, E., Rouppe van der Voort, L., Bosnjak, A., \& Antolin, P. 2013, ApJ, 774, 123

Young, P. R., Del Zanna, G., Mason, H. E., et al. 2007, PASJ, 59, S857

Young, P. R., Watanabe, T., Hara, H., \& Mariska, J. T. 2009, A\&A, 495, 587 